\title{
On unstable modules over the Dickson algebras, the Singer functors $R_{s}$ and the functors Fix
}

\author{
GEOFFREY M L POWELL
}

\begin{abstract}
The category $D_{s}-\mathcal{U}$ of unstable modules over the Steenrod algebra equipped with a compatible module structure over the Dickson algebra $D_{s}$ is studied at the prime 2, with applications to the Singer functor $R_{s}$, considered as a functor from unstable modules $U$ to $D_{s}-U$. An explicit copresentation of $R_{S} M$ is given using Lannes' $T-$ functor when $M$ is a reduced unstable module; applying Lannes' functor Fix $_{s}$, this is used to show that $R_{s}$ gives a fully-faithful embedding of $U$ in $D_{s}-U$. In addition, the right adjoint $\mathfrak{Z}_{s}$ to $R_{s}$ is introduced and is related to the indecomposables functor and the functor Fix $_{s}$.
\end{abstract}

$55 \mathrm{~S} 10 ; 18 \mathrm{E} 10$

\section{Introduction}

The Dickson algebras over the field with two elements, $\mathbb{F}$, play an important role in the theory of unstable algebras over the mod-2 Steenrod algebra; the Dickson algebra $D_{s}$ is the algebra of invariants $H^{*} V_{s}^{\operatorname{Aut}\left(V_{s}\right)}$, where $H^{*} V_{s}$ denotes the group cohomology of the rank $s$ elementary abelian 2-group $V_{s}$. The category $D_{s}-U$ of $D_{s}$-modules in the category $U$ of unstable modules arises naturally; for example, Singer introduced the functors $R_{S}$ in his work on the homology of the Steenrod algebra ([22] and related work), where $R_{s}$ can be considered as a functor from the category $U$ to $D_{s}-U$. One of the aims of this paper is to study the functor $R_{S}$, considered both as a functor to $D_{s}-U$ and as a functor to $U$, from the viewpoint of modern unstable module theory.

The functors $R_{S}$ can be applied in calculating the $E_{2}$-term of the Adams spectral sequence: Lannes and Zarati [11] related them to the derived functors of destabilization (the left adjoint to the inclusion of the category $\mathcal{U}$ in the category of graded modules over the Steenrod algebra $\mathcal{A}$ ) and these derived functors appear, via a Grothendieck spectral sequence, in the calculation of Ext groups in the category of $\mathcal{A}$-modules. The role of the Singer functors in calculating these derived functors on general modules over the Steenrod algebra has been clarified (at odd primes) by the author in [17], establishing the relationship between the approach of Lannes and Zarati [11] and that of Singer. 
For these applications, it is important to understand the behaviour of the functor $\operatorname{Hom}_{u}\left(R_{S} M,-\right)$, for $M \in \mathrm{Ob} \cup$. Restricting to the full subcategory of $U$ with objects of the form $H^{*} V$, this is equivalent to understanding $R_{s} M$ in the category $U / \mathcal{N} i l$ of unstable modules localized away from the nilpotent unstable modules, using the work of Henn, Lannes and Schwartz [8].

The paper exploits the structure of the module categories $D_{s}-U$, their relation with the categories $H^{*} V_{s}-U$ of unstable modules over $H^{*} V_{s}$ and the localized categories $D_{s}-\mathcal{U} / \mathcal{N} i l$; important tools are the functor $\mathrm{Fix}_{s}: H^{*} V_{s}-U \rightarrow U$ (see Lannes [10]) and the study of $\omega_{s}$-torsion for unstable modules over $H^{*} V_{s}$, which was initiated by Dwyer and Wilkerson [3; 4] and developed by Lannes and Zarati [12]. The latter leads to the notion of $\omega_{s}$-closure: an unstable $D_{s}$-module $M$ is $\omega_{s}$-closed if it is $\omega_{s}$-torsion-free and is maximal with this property in the equivalence class up to $\omega_{s}$-torsion.

A key new ingredient in studying the functor $R_{S}$ is an approximation $\widetilde{R}_{S}: U \rightarrow D_{s}-\mathcal{U}$ which is defined as the equalizer of a diagram

$$
D_{s} \otimes M \underset{\tau_{M}}{\stackrel{\sigma_{M}}{\longrightarrow}} H^{*} V_{s} \otimes T_{V_{s}} M
$$

in the category $D_{s}-\mathcal{U}$, where $T_{V_{s}}$ is Lannes' $T$-functor.

Theorem 1 For $s \in \mathbb{N}$, there is a natural monomorphism $\gamma_{s}: R_{S} \hookrightarrow \widetilde{R}_{S}$ of functors $u \rightarrow D_{s}-U$ such that, for an unstable module $M$,

(1) $\widetilde{R}_{S} M$ is the $\omega_{S}$-closure of $R_{S} M$;

(2) the morphism $\gamma_{s}: R_{S} M \rightarrow \widetilde{R}_{S} M$ is an isomorphism if $M$ is reduced.

This is derived from a model for the Singer functor modulo nilpotent unstable modules. The category $U / \mathcal{N} i l$ embeds in the category $\mathscr{F}$ of functors from the category $\mathscr{V} f$ of finite-dimensional $\mathbb{F}$-vector spaces to $\mathbb{F}$-vector spaces and the nillocalization of $D_{s}-\mathcal{U}$ embeds in a functor category $\mathscr{F}^{\mathfrak{g}}\left(D_{s}\right)$, for which $\mathscr{V} f$ is replaced by a category with objects $(V, W)$, where $W \leq V$ is a subspace of codimension at most $s$ in $V \in \mathrm{Ob}^{\mathscr{}} f$. The functor $R_{S}$ corresponds to the functor $\kappa_{S}: \mathscr{F}_{F} \rightarrow \mathscr{F}_{\mathfrak{g}}\left(D_{S}\right)$ given on $F \in \mathrm{Ob} \mathscr{F}_{F}$ by $\kappa_{S} F(V, W):=F(W)$. The above diagram corresponds to a copresentation of the functor $\kappa_{s}$; throughout the paper, the comparison with the behaviour after nillocalization is a guiding principle.

Theorem 1 provides a model for $R_{S} M$ (considered either in $U$ or in $D_{s}-U$ ) when $M$ is a reduced unstable module. In this case, the calculation of $T_{V} R_{S} M$ is accessible 
by using standard techniques of unstable module theory; as such, it provides a way of approaching the calculation of the functor $\operatorname{Hom}_{\mathcal{U}}\left(R_{S} M,-\right)$.

The functor $\widetilde{R}_{S}$ leads to a proof of the following result, where the natural transformation $\operatorname{Fix}_{S}\left(H^{*} V_{S} \otimes_{D_{S}} R_{S}(-)\right) \rightarrow 1_{u}$ is constructed by adjunction from the canonical monomorphism $R_{S} M \hookrightarrow D_{S} \otimes M$.

Theorem 2 For $s \in \mathbb{N}$, the natural transformation $\operatorname{Fix}_{s}\left(H^{*} V_{s} \otimes_{D_{s}} R_{S}(-)\right) \rightarrow 1_{\iota}$ of functors on $U$ is an isomorphism.

This result is striking: $\operatorname{Fix}_{S}\left(H^{*} V_{s} \otimes_{D_{s}}-\right)$ is equipped with a natural Aut $V_{S}$ action; when composed with $R_{S}$, the action is trivial. For instance, applying the functor $\mathrm{Fix}_{S}\left(H^{*} V_{S} \otimes D_{s}-\right)$ to the natural inclusion $R_{S} M \hookrightarrow D_{S} \otimes M$ yields the natural inclusion $M \hookrightarrow T_{V_{s}} M$, where $T_{V_{s}} M$ is an $\operatorname{Aut}\left(V_{s}\right)$-module by functoriality of $T_{-}$. The functor Fix $_{s}\left(H^{*} V_{s} \otimes_{D_{s}}(-)\right): D_{s}-U \rightarrow U$ can be identified in the nillocalized situation, where it corresponds to the composite functor $\Psi_{s} \operatorname{Ind}_{s}: \mathscr{F}^{\mathfrak{g}}\left(D_{s}\right) \rightarrow \mathscr{F}$, which is given on an object $G \in \mathrm{Ob} \mathscr{F}^{\mathfrak{g}}\left(D_{s}\right)$ by $\Psi_{s} \operatorname{Ind}_{s} G(V):=G\left(V \oplus \mathbb{F}^{s}, V\right)$. In this setting, Theorem 2 corresponds to the natural isomorphism $\Psi_{s} \operatorname{Ind}_{s} \kappa_{s} \cong 11_{\mathscr{F}}$ (see Lemma 7.1.6); the force of the theorem is that this lifts to unstable modules. Since the functor $\mathrm{Fix}_{S}$ does not see $\omega_{s}$-torsion, in the proof of Theorem $2, R_{S}$ can be replaced by the model $\widetilde{R}_{S}$ of Theorem 1, which leads to the result.

As a consequence, one obtains the following.

Corollary 3 The functor $R_{S}$ induces a fully-faithful embedding $R_{s}: \cup \hookrightarrow D_{s}-\mathcal{U}$, for $s \in \mathbb{N}$.

The Singer functor $R_{s}: \mathcal{U} \rightarrow D_{s}-\mathcal{U}$ admits a right adjoint $\mathfrak{Z}_{s}$. The functor $\mathfrak{Z}_{s}$ leads to a stronger conclusion (Theorem 8.3.1); Corollary 3 corresponds to the fact that the adjunction unit $1 u_{u} \rightarrow \mathfrak{Z}_{s} R_{S}$ is an isomorphism.

The functor $\mathfrak{Z}_{s}$ is of independent interest; there is a natural transformation $Q_{s} \rightarrow \mathfrak{Z}_{s}$ of functors $D_{s}-U \rightarrow \mathcal{U}$, where $Q_{s}$ is the indecomposables functor, and this is an isomorphism up to nilpotent unstable modules. After nillocalization, $Q_{s}$ corresponds to $\mathcal{R}_{s}^{0}: \mathscr{F}^{\mathfrak{g}}\left(D_{s}\right) \rightarrow \mathscr{F}_{\mathcal{F}}$ given by $\mathcal{R}_{s}^{0} G(V)=G(V, V)$; in particular the functor $Q_{s}$ becomes exact upon nillocalization.

The functor $\mathfrak{Z}_{s}$ is also related to the functor $\mathrm{Fix}_{s}$, via a natural transformation

$$
\mathfrak{Z}_{s} \rightarrow \operatorname{Fix}_{s}\left(H^{*} V_{s} \otimes_{D_{s}}(-)\right) .
$$

In the case $s=1$, this leads to a criterion (see Theorem 9.3.3) for an object of $D_{1}-U$ to be in the image of $R_{1}$. 
Organization of the paper Sections 2, 3 and 4 set the stage, providing background, introducing the categories of unstable modules over the Dickson algebras and the Singer functors respectively. Sections 5 and 6 introduce the tools of nillocalization as they apply to unstable modules over Dickson algebras and Section 7 gives the model for the Singer functors viewed through the filter of nillocalization.

The main results of the paper are proved in Sections 8 and 9.

\section{Background}

\subsection{Unstable modules and unstable algebras}

Throughout the paper, $\mathbb{F}$ is the field with two elements and $\mathcal{A}$ is the mod-2 Steenrod algebra (see Schwartz [19] for the basics of the theory of unstable modules over the Steenrod algebra). The category of graded $\mathcal{A}$-modules is denoted by $\mathcal{M}$ and the full subcategory of unstable modules $U$; these are equipped with the usual tensor product. A commutative algebra $B$ in $M$ is unstable if the underlying module is unstable and satisfies $\mathrm{Sq}_{0} x=x^{2}$, where $\mathrm{Sq}_{0}$ denotes the top Steenrod operation; the category of unstable algebras and algebra morphisms is denoted by $\mathscr{K}$. Observe that the degree zero part of an unstable algebra is a Boolean algebra. An unstable algebra is Noetherian if the underlying commutative algebra is finitely-generated.

The category of $B$-modules in $\mathcal{M}$ is denoted by $B-\mathcal{M}$ and, if $K$ is an unstable algebra, the category of $K$-modules in $U$ is denoted by $K-\mathcal{U}$. If $K \rightarrow L$ is a morphism of unstable algebras, there is an adjunction

$$
L \otimes_{K}-: K-U \rightleftarrows L-U: \operatorname{Restrict}_{K}^{L},
$$

where $L \otimes_{K}-$ is the induction functor, left adjoint to the exact restriction functor.

The degree-doubling functor $\Phi: \mathcal{M} \rightarrow \mathcal{M}$ restricts to a functor $\Phi: \mathcal{U} \rightarrow \mathcal{U}$ and $\mathrm{Sq}_{0}$ induces a natural transformation $\lambda: \Phi \rightarrow 1_{u}$ (see [19, Section 1.7]). An unstable module $M$ is reduced if $\lambda_{M}$ is a monomorphism (equivalently, if $M$ does not contain a nilpotent submodule, where an unstable module $N$ is nilpotent if $\mathrm{Sq}_{0}$ acts locally nilpotently); $M$ is nilclosed if $\operatorname{Ext}_{{ }^{\varepsilon}}^{\varepsilon}(N, M)=0$, for every $\varepsilon \in\{0,1\}$ and nilpotent $N$. The full subcategory of nilpotent unstable modules $\mathcal{N} i l \subset \mathcal{U}$ is a localizing subcategory (see Gabriel [6] for generalities on localization of abelian categories).

An unstable algebra is reduced (resp. nilclosed) if the underlying unstable module has this property, hence an unstable algebra $K$ is reduced if and only if $K$ contains no nilpotent elements. The functor $\Phi$ commutes with tensor products, thus restricts to a functor $\Phi: \mathscr{K} \rightarrow \mathscr{K}$, and $\Phi$ induces an exact functor $\Phi: K-\mathcal{U} \rightarrow \Phi K-\mathcal{U}$. If $K$ 
is reduced, $\Phi K$ identifies via $\lambda_{K}$ as the unstable subalgebra of $K$ generated by the squares of elements of $K$.

The functor $\widetilde{\Phi}: \mathcal{U} \rightarrow \mathcal{U}$ is the right adjoint to $\Phi$ (see [19, Examples 2.2.3]) and the adjunction unit $M \rightarrow \widetilde{\Phi} \Phi M$ is a natural isomorphism. Proposition A.1.1 shows that $\widetilde{\Phi}$ induces a right adjoint to $\Phi: K-\vartheta \rightarrow \Phi K-\vartheta$.

\subsection{Lannes' $T$-functor}

For $V$ an elementary abelian 2-group ( $V_{s}$ will be written to denote an elementary abelian 2-group of rank $s), H^{*} V$ denotes the group cohomology of $V$ with $\mathbb{F}-$ coefficients, which is isomorphic to the symmetric algebra $S^{*}\left(V^{*}\right)$ on the dual of $V$; the underlying unstable module of $H^{*} V$ is injective in $U$ (see [19, Chapter 3]).

Lannes' $T$-functor $T_{V}: \cup \rightarrow \mho$ is the left adjoint to $H^{*} V \otimes-: ~ U \rightarrow \vartheta$; it is exact and commutes with tensor products. Moreover, $T_{V}$ restricts to a functor $T_{V}: \mathscr{K} \rightarrow \mathscr{K}$ and, for an unstable algebra $K$, induces an exact functor $K-\vartheta \rightarrow T_{V} K-\mathcal{u}$.

A morphism of unstable algebras $\varphi: K \rightarrow H^{*} V$ is adjoint to a morphism $T_{V} K \rightarrow \mathbb{F}$ of unstable algebras, which factors across a morphism of Boolean algebras $\tilde{\varphi}: T_{V}^{0} K \rightarrow \mathbb{F}$, where $T_{V}^{0}$ denotes the degree zero part of $T_{V} ; \mathbb{F}$ is a flat $T_{V}^{0} K$-module with respect to this morphism, so that $\mathbb{F} \otimes T_{V}^{0} K-$ is exact on the category of $T_{V}^{0} K$-modules.

Definition 2.2.1 For $\varphi: K \rightarrow H^{*} V$ a morphism of unstable algebras, let $T_{(V, \varphi)} K$ denote the unstable algebra $\mathbb{F} \otimes T_{V}^{0} K T_{V} K$, where $\mathbb{F}$ is a $T_{V}^{0} K$-algebra via $\tilde{\varphi}$, and let $T_{(V, \varphi)}: K-\vartheta \rightarrow T_{(V, \varphi)} K-\vartheta$ be the exact functor $\mathbb{F} \otimes_{T_{V}^{0} K} T_{V}(-)$.

The functor $T_{V}$ is natural in $V$; in particular, there is a natural inclusion $1_{u} \cong T_{0} \hookrightarrow T_{V}$, for $V \in \mathrm{Ob}^{f}$.

Lemma 2.2.2 For $K \in \mathrm{Ob} \mathcal{K}, M \in \mathrm{Ob} K-\mathcal{U}$ and $\varphi: K \rightarrow H^{*} V$ a morphism of unstable algebras, there are morphisms of unstable algebras

$$
K \rightarrow T_{V} K \rightarrow T_{(V, \varphi)} K \cong \mathbb{F} \otimes_{T_{V}^{0} K} T_{V} K
$$

with respect to which the natural morphisms of unstable modules

$$
M \rightarrow T_{V} M \rightarrow T_{(V, \varphi)} M \cong \mathbb{F} \otimes_{T_{V}^{0} K} T_{V} M
$$

are morphisms of $K-\boldsymbol{U}$. 


\subsection{The Dickson algebras}

The group $\operatorname{Aut}(V)$ of linear automorphisms acts on $H^{*} V$ on the right by morphisms of unstable algebras; the Dickson algebra $D_{V} \in \mathrm{Ob} \mathscr{K}$ is the ring of invariants $H^{*} V^{\operatorname{Aut}(V)}$; $D_{V_{s}}$ will be denoted by $D_{s}$. There is an isomorphism of graded algebras

$$
D_{s} \cong \mathbb{F}\left[\omega_{s, 0}, \ldots, \omega_{s, s-1}\right],
$$

where the generator $\omega_{s, i}$ has degree $2^{s}-2^{i}$; see Wilkerson [25]. The top Dickson invariant $\omega_{s, 0}$ will be written $\omega_{s}$ and identifies with the product of the elements of $\left(H^{1} V_{S}\right) \backslash\{0\}$; there are related explicit descriptions of the other generators. The algebra $H^{*} V_{s}$ is free as a $D_{s}$-module, forgetting the action of the Steenrod algebra (see Neusel and Smith [16] for example), in particular is flat as a $D_{s}$-module.

Lemma 2.3.1 [11, Définition-Proposition 4.4.7] Let $\alpha: U \hookrightarrow V$ be the inclusion of a subspace of codimension $c$. There is a canonical surjection of unstable algebras $D_{V} \rightarrow \Phi^{c} D_{U}$ which fits into a commutative diagram

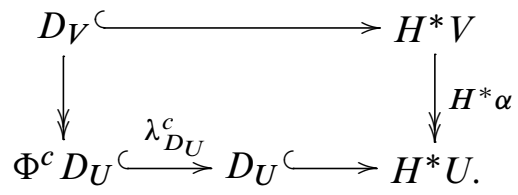

In particular, for $s \in \mathbb{N}$, the kernel of $D_{s} \rightarrow \Phi D_{s-1}$ is the prime ideal $\omega_{s} D_{s}$, which is invariant under the $\mathcal{A}$-action.

The Boolean algebra $T_{W}^{0} H^{*} V_{s}$ identifies with $\mathbb{F}^{\mathrm{Hom}\left(W, V_{s}\right)}$, and by [19, Proposition 3.9.8], the subalgebra $T_{W}^{0} D_{s}$ is isomorphic to $\mathbb{F}^{\operatorname{Hom}\left(W, V_{s}\right) / \operatorname{Aut}\left(V_{s}\right)}$. The mor-

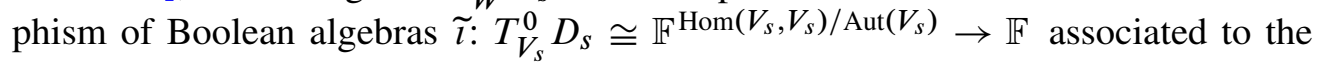
canonical inclusion $i$ : $D_{S} \hookrightarrow H^{*} V_{S}$ is induced by evaluation on the element of $\operatorname{Hom}\left(V_{s}, V_{s}\right) / \operatorname{Aut}\left(V_{s}\right)$ represented by the identity morphism of $V_{s}$.

Proposition 2.3.2 For $s \in \mathbb{N}$,

(1) the unstable algebra $T_{\left(V_{s}, i\right)} D_{s}$ is isomorphic to $H^{*} V_{s}$;

(2) $T_{\left(V_{s}, i\right)}$ induces an exact functor $T_{\left(V_{s}, i\right)}: D_{s}-U \rightarrow H^{*} V_{s}-U$.

Proof The first statement is a case of the calculation of the $T$-functor on rings of invariants (cf [19, Proposition 3.9.8], Dwyer and Wilkerson [5, proof of 1.4]). The second is an immediate consequence, following from the definition of $T_{\left(V_{s}, i\right)}$. 


\subsection{Unstable modules over $H^{*} V_{s}$ and $\mathrm{Fix}_{s}$}

The module category $D_{s}-U$ is related to the category $H^{*} V_{s}-U$ via the adjunction

$$
H^{*} V_{s} \otimes D_{s}-: D_{s}-U \rightleftarrows H^{*} V_{s}-U: \operatorname{Restrict}_{D_{s}}^{H^{*} V_{s}} .
$$

The functor $\mathrm{Fix}_{s}: H^{*} V_{s}-\mathcal{U} \rightarrow \mathcal{U}$ is the left adjoint to the free $H^{*} V_{s}$-module functor $H^{*} V_{s} \otimes-:$ U $\rightarrow H^{*} V_{s}-U$; it commutes with tensor products and restricts to a functor Fix $_{s}: H^{*} V_{s} \downarrow \mathscr{K} \rightarrow \mathscr{K}$ which is left adjoint to $H^{*} V_{s} \otimes-: \mathscr{K} \rightarrow H^{*} V_{s} \downarrow \mathscr{K}$ (cf [12, Théorème 1.3.3]). See [10] and [12;13] for further properties of the categories $H^{*} V_{S}-\Theta$ and the functors Fix $_{s}$.

Lemma 2.2.2 has the following analogue for the functor Fix ${ }_{s}$, using Lannes' description of Fix $_{s}$ in terms of $T_{V_{s}}$.

Proposition 2.4.1 For $s \in \mathbb{N}$, the natural transformation $1_{u} \rightarrow T_{V_{s}}$ induces a natural transformation Forget $\rightarrow$ Fix $_{s}$, where Forget ${ }_{s}: H^{*} V_{s}-U \rightarrow U$ is the forgetful functor; this factors naturally

$$
\text { Forget }_{S} \longrightarrow \mathbb{F} \otimes H^{*} V_{s}(-) \longrightarrow \text { Fix }_{S}(-)
$$

across the $H^{*} V_{s}$-module indecomposables.

Proof By [10, 4.4.3], Fix $M \cong \mathbb{F} \otimes{T_{V_{s}} H^{*} V_{s}}_{V_{V_{s}}} M$, where $T_{V_{s}} H^{*} V_{s} \rightarrow \mathbb{F}$ is adjoint to the identity on $H^{*} V_{s}$. The natural morphism $M \rightarrow T_{V_{s}} M$ defines a morphism of $H^{*} V_{s}$-modules, as in Lemma 2.2.2. The $H^{*} V_{s}$-module structure on $\operatorname{Fix}_{s} M$ is induced by the morphism of unstable algebras $H^{*} V_{s} \rightarrow \operatorname{Fix}_{s} H^{*} V_{s} \cong \mathbb{F}$. The result follows.

\section{Unstable modules over the Dickson algebras}

\subsection{Unstable modules over $D_{s}, \omega_{s}$-torsion and $\omega_{s}-$ closure}

Recall from Lemma 2.3.1 that there is a canonical surjection of unstable algebras $D_{s} \rightarrow \Phi D_{s-1}$; this induces the functors in the following standard result.

Proposition 3.1.1 For $s \in \mathbb{N}$, there are adjunctions

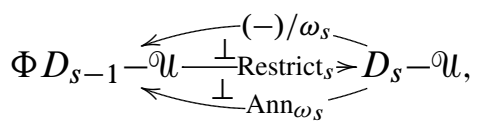

where (-)/ $\omega_{s}: M \mapsto M / \omega_{s} M$, for $M \in \mathrm{Ob} D_{s}-\mathcal{U}$ and $\mathrm{Ann}_{\omega_{s}} M$ is the submodule of elements $x$ such that $\omega_{s} x=0$. 
Moreover, the adjunction Restrict ${ }_{s} \dashv \mathrm{Ann}_{\omega_{s}}$ identifies $\Phi D_{s-1}-\mathcal{U}$ as the full subcategory of $D_{s}-U$ of modules annihilated by $\omega_{s}$.

Remark 3.1.2 (1) The functor $(-) / \omega_{s}$ identifies with $\Phi D_{s-1} \otimes_{D_{s}}-$, the induction functor.

(2) Localization away from the torsion associated to an invariant ideal of an unstable algebra has been considered by Henn [7, Section 3] and Meyer [15, Chapter 7]. Dwyer and Wilkerson [3; 4] and Lannes and Zarati [12] have considered localization away from $\omega_{s}$-torsion.

Localization inverting the top Dickson invariant is an important tool. For $s \in \mathbb{N}$, the localized algebras $D_{s}\left[\omega_{s}^{-1}\right]$ and $H^{*} V_{s}\left[\omega_{s}^{-1}\right]$ are commutative algebras in $M$ (see Singer [21] and Wilkerson [24]); moreover, $D_{s}\left[\omega_{s}^{-1}\right] \otimes_{D_{s}}-$ induces an exact functor

$$
D_{s}\left[\omega_{s}^{-1}\right] \otimes_{D_{s}}-: D_{s}-u \rightarrow D_{s}\left[\omega_{s}^{-1}\right]-\mu,
$$

which will be denoted $M \mapsto M\left[\omega_{s}^{-1}\right]$.

Recall that the inclusion $U \hookrightarrow \mathcal{M}$ has a right adjoint Un: $\mathcal{M} \rightarrow \mathcal{U}$, which gives the largest unstable module of an $\mathcal{A}$-module. If $X, Y$ are $\mathcal{A}$-modules, there is a canonical monomorphism $(\mathrm{Un} X) \otimes(\mathrm{Un} Y) \rightarrow \mathrm{Un}(X \otimes Y)$. It follows that, for $M \in \mathrm{Ob} D_{s}-\mathcal{U}$, there is a natural morphism $M \rightarrow \operatorname{Un}\left(M\left[\omega_{s}^{-1}\right]\right)$ in $D_{s}-U$.

Definition 3.1.3 $[7 ; 15]$ An unstable $D_{s}$-module $M \in \mathrm{Ob} D_{s}-\mathcal{U}$ is $\omega_{s}$-closed if the map $M \rightarrow \operatorname{Un}\left(M\left[\omega_{s}^{-1}\right]\right)$ is an isomorphism. An unstable $H^{*} V_{s}$-module is $\omega_{s}$-closed if the underlying unstable $D_{s}$-module is $\omega_{s}$-closed.

Proposition 3.1.4 For $s \in \mathbb{N}$, an unstable $D_{s}$-module $M$ is $\omega_{s}$-closed if the unstable $H^{*} V_{s}$-module $H^{*} V_{s} \otimes_{D_{s}} M$ is $\omega_{s}$-closed.

In particular, for $N \in \mathrm{Ob} U, D_{s} \otimes N$ is $\omega_{s}$-closed.

Proof The unstable $D_{s}$-module $M$ embeds in $H^{*} V_{s} \otimes_{D_{s}} M$ as the invariants (in $\left.D_{s}-U\right)$ of the action of $\operatorname{Aut}\left(V_{s}\right)$ induced by the action on the left hand factor. This implies that, if $H^{*} V_{s} \otimes D_{s} M$ is $\omega_{s}$-closed, then $M$ is $\omega_{s}$-closed, since the kernel of a morphism between $\omega_{S}$-closed objects is $\omega_{s}$-closed.

By [11, Proposition 2.5.2], the morphism

$$
H^{*} V_{s} \otimes N \rightarrow \operatorname{Un}\left(H^{*} V_{s}\left[\omega_{s}^{-1}\right] \otimes N\right)
$$

is an isomorphism, for $N$ an unstable module, which shows that $H^{*} V_{s} \otimes N$ is $\omega_{s}-$ closed. Since $H^{*} V_{s} \otimes N$ is isomorphic to $H^{*} V_{s} \otimes D_{s}\left(D_{s} \otimes N\right)$, it follows that $D_{s} \otimes N$ is $\omega_{s}$-closed. 
Remark 3.1.5 The converse is false in general. Consider $H^{*} V_{s}$ as a $D_{s}$-module (which is $\omega_{s}$-closed); for $s>1$ an integer, $H^{*} V_{s} \otimes_{D_{s}} H^{*} V_{s}$ is not $\omega_{s}$-closed.

\subsection{The indecomposables functor}

The augmentation $D_{s} \rightarrow \mathbb{F}$ induces a functor triv ${ }_{s}: U \rightarrow D_{s}-\mathcal{U}$ which has left adjoint $Q_{s}: D_{s}-U \rightarrow \mathcal{U}$ given by $M \mapsto M / \overline{D_{s}} M \cong \mathbb{F} \otimes_{D_{s}} M, \overline{D_{s}}$ denoting the augmentation ideal, and right adjoint which associates to an unstable $D_{s}$-module the largest unstable submodule with trivial $D_{s}$-action, thus identifying $U$ as the full subcategory of $D_{s}-\mathcal{U}$ of objects with trivial $D_{s}$-module structure

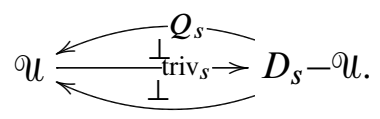

\section{Introducing the Singer functors}

\subsection{The Singer functor $R_{s}$}

The definition and properties of the Singer functor $R_{s}: U \rightarrow D_{s}-U$ are reviewed in this section; for further details, the reader is referred to the original work of Singer [23; 20] (and related work) and Lannes and Zarati's paper [11].

In the following, if $I$ is a sequence of nonnegative integers, $S q^{I}$ denotes the Milnor basis element of the Steenrod algebra indexed by $I$. The linear map $\mathrm{St}_{s}$ introduced below corresponds to the Steenrod total power.

Definition 4.1.1 For $s \geq 1$ an integer and $M$ an unstable module, let

(1) $\mathrm{St}_{s}: \Phi^{s} M \rightarrow D_{s} \otimes M$ be the linear map defined on a homogeneous element $\Phi^{s} x$ by

$$
\mathrm{St}_{s}\left(\Phi^{s} x\right):=\sum_{I=\left(i_{1}, \ldots, i_{s}\right)} \omega_{s, 0}^{\varepsilon} \omega_{s, 1}^{i_{1}} \ldots \omega_{s, s-1}^{i_{s-1}} \otimes S q^{I}(x)
$$

where $|x|=\varepsilon+i_{1} \cdots+i_{s}$;

(2) $R_{s} M$ denote the sub $D_{s}$-module (ignoring the $\mathcal{A}$-action) of $D_{s} \otimes M$ which is generated by the image of $\mathrm{St}_{s}$.

Remark 4.1.2 (1) The linear maps $\mathrm{St}_{s}$ can be constructed as iterates of the linear maps $\mathrm{St}_{1}$; the above definition stresses the intimate relationship between the Dickson algebras and the dual Steenrod algebra. 
(2) Proposition 4.1.3 below contains the statement that $R_{s} M \subset D_{s} \otimes M$ is stable under the action of the Steenrod algebra, which is not immediately obvious from the definition given.

By convention, $R_{0}$ is taken to be the identity functor on $U$ and $R_{-1}$ to be the zero functor.

Proposition 4.1.3 [11] For $s \in \mathbb{N}, R_{s}$ defines a functor $R_{s}: \mathcal{U} \rightarrow D_{s}-\mathcal{U}$, equipped with a monomorphism $R_{s}(-) \hookrightarrow D_{s} \otimes-$ in $D_{s}-U$.

(1) For $M$ an unstable module, the underlying graded $D_{s}$-module of $R_{S} M$ is free on a vector space isomorphic to $\Phi^{s} M$. Moreover, there is a natural isomorphism $Q_{s} R_{s} M \cong \mathbb{F} \otimes D_{s} R_{s} M \cong \Phi^{s} M$ in $u$.

(2) The functor $R_{s}: \cup \rightarrow D_{s}-\mathcal{U}$ is exact and commutes with limits and colimits.

(3) For unstable modules $M, N$, there is a natural isomorphism $R_{S}(M \otimes N) \cong$ $R_{s} M \otimes_{D_{s}} R_{s} N$ in $D_{s}-U$.

(4) There is a natural surjection $\rho_{s}: R_{s} M \rightarrow \Phi R_{s-1} M$ in $D_{s}-u$ which makes the following diagram commute:

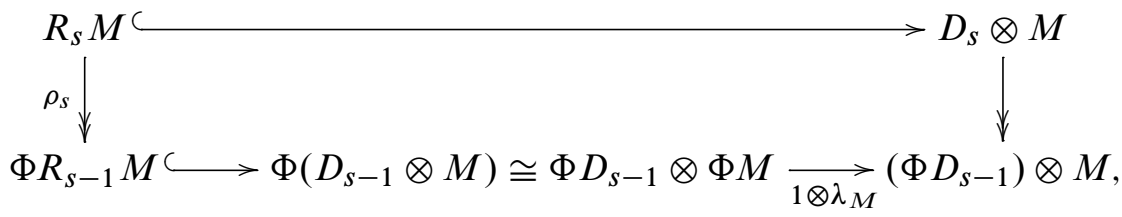

where the terms of the bottom row are considered as $D_{s}-$ modules via restriction of their natural $\Phi D_{s-1}-$ module structures.

Moreover, there is a natural short exact sequence in $D_{s}-U$,

$$
0 \rightarrow \omega_{s} R_{s} M \rightarrow R_{s} M \rightarrow \Phi R_{s-1} M \rightarrow 0
$$

(5) If $N$ is a nilpotent unstable module, then $R_{S} N$ is nilpotent.

(6) If $M$ is a reduced (respectively nilclosed) unstable module, then $R_{S} M$ is reduced (resp. nilclosed).

The following result will be strengthened in Section 8.3.

Lemma 4.1.4 For $s \in \mathbb{N}$ and unstable modules $M, N$, the functor $R_{s}$ induces a monomorphism $\operatorname{Hom}_{u}(M, N) \hookrightarrow \operatorname{Hom}_{D_{s}-\mathcal{U}}\left(R_{S} M, R_{S} N\right)$. 
Proof By Proposition 4.1.3, the composite $Q_{s} R_{S}$ is naturally isomorphic to the functor $\Phi^{s}$, hence gives rise to

$$
\operatorname{Hom}_{u}(M, N) \stackrel{R_{s}}{\longrightarrow} \operatorname{Hom}_{D_{s}-U}\left(R_{S} M, R_{S} N\right) \stackrel{Q_{s}}{\longrightarrow} \operatorname{Hom}_{U}\left(\Phi^{s} M, \Phi^{s} N\right),
$$

which identifies with the natural morphism corresponding to the functor $\Phi^{s}$. The functor $\Phi^{s}$ is fully faithful, hence the first morphism is injective, as required.

Recall that an unstable module $N$ is locally finite if $\mathcal{A} x \subset N$ is finite, for every element $x$ of $N$.

Proposition 4.1.5 For $s \in \mathbb{N}$ and $X$ a locally finite unstable module, the natural monomorphism $R_{S} X \hookrightarrow D_{S} \otimes X$ is the $\omega_{s}$-closure of $R_{S} X$ in $D_{s} \otimes X$.

Proof The module $D_{s} \otimes X$ is $\omega_{s}$-closed in $D_{s}-U$, by Proposition 3.1.4, hence it suffices to show that the cokernel of $R_{S} X \rightarrow D_{s} \otimes X$ is $\omega_{s}$-torsion. Since both functors commute with colimits, it suffices to consider the case where $X$ is a finite unstable module. This case is established by induction on the total dimension of $X$, using the cases $X=\Sigma^{n} \mathbb{F}$, for $n \in \mathbb{N}$, for the inductive step. The monomorphism $R_{s} \Sigma^{n} \mathbb{F} \hookrightarrow D_{s} \otimes \Sigma^{n} \mathbb{F}$ identifies with the $n$-iterated suspension of the inclusion $\omega_{s}^{n} D_{s} \hookrightarrow D_{s}$, so the cokernel is $\omega_{s}$-torsion.

Proposition 4.1.6 The functor $R_{s}: \cup \rightarrow D_{s}-\mathcal{U}$ admits a left adjoint $\mathfrak{A}_{s}$ and a right adjoint $\mathfrak{Z}_{s}$,

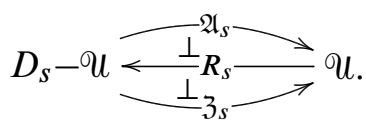

Moreover,

(1) the functor $\mathfrak{A}_{s}$ sends projective objects of $D_{s}-\mathfrak{U}$ to projectives of $\mathscr{U}$;

(2) the functor $\mathfrak{Z}_{s}$ sends injective (respectively reduced) objects of $D_{s}-u$ to injective (resp. reduced) objects of $u$.

Proof The result is a formal consequence of the properties of $R_{S}$. For example, for $M \in \mathrm{Ob} D_{s}-u, \mathfrak{Z}_{s} M$ is reduced if and only if $\operatorname{Hom}_{u}\left(N, \mathfrak{Z}_{s} M\right) \cong \operatorname{Hom}_{D_{S}-u}\left(R_{S} N, M\right)$ is trivial for every nilpotent unstable module $N$. The functor $R_{S}$ preserves nilpotent unstable modules; hence, if $M$ is reduced, then $\operatorname{Hom}_{D_{S}-\Omega}\left(R_{S} N, M\right)=0$ for nilpotent $N$. 
Remark 4.1.7 The category $D_{s}-\mathcal{U}$ has enough projectives and injectives [12; 15]. A family of projective generators of $D_{s}-\mathcal{U}$ is given by the family of unstable $D_{s}$-modules $D_{s} \otimes F(n)$, where $F(n)$ denotes the free unstable module on a generator of degree $n$. Similarly, there is a family of injective cogenerators given by the generalized BrownGitler modules $J_{D_{s}}(n)$, for $n \in \mathbb{N}$, where $J_{D_{s}}(n)$ corepresents the contravariant functor $M \mapsto\left(M^{n}\right)^{*}$, for $M \in \mathrm{Ob} D_{s}-\mathcal{U}$.

The unstable modules $\mathfrak{A}_{s}\left(D_{s} \otimes F(n)\right)$ and $\mathfrak{Z}_{s}\left(J_{D_{s}}(n)\right)$ are closely related and illuminate the relationship between the Dickson algebras and the Steenrod algebra.

Recall from Section 3 that $\operatorname{triv}_{s}: \mathcal{U} \rightarrow D_{s}-\mathcal{U}$ gives an unstable module the trivial $D_{s}$-module structure and Restrict ${ }_{s}: \Phi D_{s-1}-\mathcal{U} \rightarrow D_{s}-\mathcal{U}$ is induced by the canonical projection $D_{s} \rightarrow \Phi D_{s-1}$. The functor $\widetilde{\Phi}: \Phi D_{s-1}-U \rightarrow D_{s-1}-\mathcal{U}$ is provided by Proposition A.1.1.

Proposition 4.1.8 For $s \in \mathbb{N}$, there are natural isomorphisms

(1) $\mathfrak{Z}_{s} \circ$ Restrict $_{s} \cong \mathfrak{Z}_{s-1} \circ \widetilde{\Phi}: \Phi D_{s-1}-\mathcal{U} \rightarrow \boldsymbol{U}$

(2) $\mathfrak{Z}_{s} \circ \operatorname{triv}_{s} \cong \widetilde{\Phi}^{s}: \cup \rightarrow$ U.

Proof For $M \in \mathrm{Ob} U$ and $N \in \mathrm{Ob} \Phi D_{s-1}-\mathcal{U}$, there are natural isomorphisms

$$
\begin{aligned}
\operatorname{Hom}_{U}\left(M, \mathfrak{Z}_{S} \operatorname{Restrict}_{S} N\right) & \cong \operatorname{Hom}_{D_{S}-u}\left(R_{S} M, \operatorname{Restrict}_{S} N\right) \\
& \cong \operatorname{Hom}_{\Phi D_{s-1}-\imath}\left(\left(R_{S} M\right) / \omega_{s}, N\right) .
\end{aligned}
$$

By Proposition 4.1.3, there is a natural isomorphism $\left(R_{S} M\right) / \omega_{s} \cong \Phi R_{S-1} M$, hence $\operatorname{Hom}_{\Phi D_{s-1}-u}\left(\left(R_{s} M\right) / \omega_{s}, N\right) \cong \operatorname{Hom}_{D_{s-1}-u}\left(R_{s-1} M, \widetilde{\Phi} N\right) \cong \operatorname{Hom}_{U}\left(M, \mathfrak{Z}_{s-1} \tilde{\Phi} N\right)$, by adjunction. The first statement follows.

The second statement can either be proved directly by a similar argument, or deduced by induction from the first, since $\operatorname{triv}_{s}$ is the composite of the functors Restrict ${ }_{i}$ for $1 \leq i \leq s$.

Further results on the functors $\mathfrak{Z}_{s}$ are given in Section 9, using deeper properties of the Singer functors $R_{S}$.

\section{Functor categories and nillocalization}

This section reviews the techniques of nillocalization, as they apply to the study of the category of unstable modules over an unstable algebra. This is based on the foundations of Henn, Lannes and Schwartz [8; 9], related to earlier work of Lam, Rector [18] and Adams and Wilkerson [1], and on subsequent work of Djament [2], Henn [7], Lannes and Zarati [12; 13], Mekkia [14] and Meyer [15]. 


\subsection{Nillocalizations}

The general theory of localization of abelian categories [6] provides an adjunction $l: \mathcal{U} \rightleftarrows U / \mathcal{N} i l: r$ and, moreover, the functor $l$ is exact [19, Chapter 5]. The adjunction unit $M \rightarrow r l M$ corresponds to nilclosure: $M$ is reduced (respectively nilclosed) if and only if it is a monomorphism (resp. isomorphism).

Notation 5.1.1 Write $\mathscr{V} f$ for the full subcategory of finite-dimensional spaces in $\mathscr{V}$, the category of $\mathbb{F}$-vector spaces; the category of functors from $\mathscr{V} f$ to $\mathscr{V}$ is denoted by $\mathscr{F}_{F}$ and the full subcategory of locally finite (or analytic) functors, $\mathscr{F}_{\omega}$ (see $[8 ; 19]$ ).

The category $\mathcal{U} / \mathcal{N} i l$ identifies with the full subcategory $\mathscr{F}_{\omega} \subset \mathscr{F}_{\text {of }}$ analytic functors via $l: U \rightarrow \mathscr{F}, l M(V)=T_{V}^{0} M$, which has right adjoint equally denoted by $r$ :

$$
l: U \rightleftarrows \mathscr{F}: r .
$$

Example 5.1.2 For $V \in \mathrm{Ob}^{\mathcal{V}} f$, we have that the analytic functor $l H^{*} V$ is the injective $I_{V}(-)=\mathbb{F}^{\mathrm{Hom}(-, V)}$. The analogue of the functor $T_{V}$ in the category $\mathscr{F}$ is the shift functor $\Delta_{V}: \mathscr{F} \rightarrow \mathscr{F}$, defined by precomposition with $-\oplus V: \mathscr{V} f \rightarrow \mathscr{V} f$.

The functors $l$ and $r$ both commute with tensor products, which is an important fact in considering module structures in the respective categories. Similarly, the functor $l$ sends an unstable algebra to a functor with values in Boolean algebras. The category of Boolean algebras is equivalent to the opposite of the category of profinite sets, via the functor $X \mapsto \mathbb{F}^{X}$, where $\mathbb{F}^{X}$ denotes the space of continuous maps from the profinite set $X$ to $\mathbb{F}$.

\section{Notation 5.1.3 Let}

(1) $\mathscr{P} \mathscr{S}$ denote the category of presheaves of profinite sets on $\mathscr{q}^{f}$, so that the continuous map functor induces $\mathbb{F}^{(-)}: \mathscr{P} \mathscr{S}^{\circ p} \rightarrow \mathscr{F}$;

(2) $\mathfrak{g}: \mathscr{K}^{\mathrm{op}} \rightarrow \mathscr{P} \mathscr{Y}$ be the functor $\mathfrak{g}(K): V \mapsto \operatorname{Hom}_{\mathscr{K}}\left(K, H^{*} V\right)$.

Lannes' linearization principle fits into this framework via the isomorphism

$$
l(K)(V)=T_{V}^{0} K \cong \mathbb{F}^{\mathfrak{g}(K)(V)} .
$$

If $K$ is a Noetherian unstable algebra, then $\mathfrak{g}(K)$ takes values in finite sets (cf [8]). 
Example 5.1.4 For $s \in \mathbb{N}$,

(1) $\mathfrak{g}\left(H^{*} V_{s}\right)(W)=\operatorname{Hom}_{\mathscr{V} f}\left(W, V_{s}\right)$;

(2) $\mathfrak{g}\left(D_{s}\right)(W)=\operatorname{Hom}_{\mathscr{V} f}\left(W, V_{s}\right) / \operatorname{Aut}\left(V_{s}\right)$, which is equivalent to the set of subspaces of $W$ of codimension at most $s$, regarded as a contravariant functor by pullback of subspaces.

The inclusion $D_{s} \stackrel{i}{\hookrightarrow} H^{*} V_{s}$ induces the surjection to coinvariants $\mathfrak{g}\left(H^{*} V_{S}\right) \rightarrow \mathfrak{g}\left(D_{S}\right)$.

\subsection{Nillocalization of the category of modules over a Noetherian unstable algebra}

Let $K$ be a Noetherian unstable algebra; an object of $K-U$ is said to be nilpotent if the underlying unstable module is nilpotent. There is an exact localization functor $K-U \rightarrow K-U / \mathcal{N} i l$. (This notation should not lead to confusion, since there is a forgetful functor to $U / \mathcal{N} i l$ and the category $K-\mathcal{U} / \mathcal{N} i l$ only depends on $K$ up to nillocalization.)

An element of $K-U$ is nilclosed if and only if the underlying unstable module is nilclosed; in this case, the unstable $K$-module structure is the restriction of the induced unstable $r l(K)$-module structure $(r l(K)$ has a canonical unstable algebra structure [8]).

An element $\varphi \in \mathfrak{g}(K)(V)$ can be considered as a morphism of Boolean algebras $T_{V}^{0} K \rightarrow \mathbb{F}$ and the functor $T_{(V, \varphi)}$ is defined (cf Definition 2.2.1), which has degree zero part denoted $T_{(V, \varphi)}^{0}$. The pair $(V, \varphi)$ can be considered as an object of a comma category, which motivates the following.

Notation 5.2.1 For $\mathfrak{X}$ a presheaf of finite sets on $\mathfrak{V}^{f}$, denote by

(1) $\mathscr{V}_{/ \mathfrak{X}}^{f}$ the comma category, with objects pairs $(V, x)$, where $V \in \mathscr{V} f$ and $x \in \mathfrak{X}(V)$, and a morphism $(V, x) \rightarrow(W, y)$ is a linear map $f: V \rightarrow W$ such that $\mathfrak{X}(f) y=x$;

(2) $\mathscr{F}^{\mathfrak{X}}$ the category of functors $\operatorname{Funct}\left(\mathscr{V}_{/ \mathfrak{X}}^{f}, \mathscr{V}\right)$;

(3) $\mathbb{F}^{\mathfrak{X}}-\mathscr{F}$ the category of $\mathbb{F}^{\mathfrak{X}}$-modules in $\mathscr{F}_{\text {. }}$

Example 5.2.2 (1) The category $\mathscr{V}_{/ \mathfrak{g}\left(H^{*} V_{S}\right)}^{f}$ is the over-category $\mathscr{V}^{f} / V_{s}$.

(2) The category $\mathscr{V}_{/ \mathfrak{g}\left(D_{s}\right)}^{f}$ has objects $(V, U)$, where $U \leq V$ is a subspace of codimension at most $s$; a morphism $(V, U) \rightarrow\left(V^{\prime}, U^{\prime}\right)$ is a linear map $V \rightarrow V^{\prime}$ sending $U$ to $U^{\prime}$ and such that the induced map $V / U \rightarrow V^{\prime} / U^{\prime}$ is a monomorphism. 
The category $\mathscr{F}^{\mathfrak{X}}$ is abelian equipped with a tensor product, this structure being inherited from $\mathscr{V}$. Moreover, Yoneda's lemma shows that it has sufficiently many projectives and injectives.

Example 5.2.3 Consider the case $\mathfrak{X}=\mathfrak{g}\left(D_{s}\right)$, for $s \in \mathbb{N}$. If $(V, U)$ is an object of $q_{/ \mathfrak{g}\left(D_{s}\right)}^{f}$, then

(1) $P_{(V, U)} \in \mathrm{Ob} \mathscr{F} \mathfrak{g}\left(D_{s}\right)$ denotes the projective functor $\mathbb{F}[\operatorname{Hom}((V, U),-)]$,

(2) $I_{(V, U)} \in \mathrm{Ob} \mathscr{F}^{\mathfrak{g}\left(D_{s}\right)}$ denotes the injective functor $\mathbb{F}^{\mathrm{Hom}(-,(V, U))}$,

where Hom is taken in the category $\mathscr{V}_{/ \mathfrak{g}\left(D_{s}\right)}^{f}$. This gives families of projective generators and injective cogenerators respectively, as $(V, U)$ runs over representatives of isomorphism classes of objects of $\mathscr{V}_{/ \mathfrak{g}\left(D_{s}\right)}^{f}$.

The functor $I_{\left(V_{s}, 0\right)}$ plays an important role; it can be identified as follows. There is a natural isomorphism

$$
\operatorname{Hom}_{\mathscr{v}_{/ \mathfrak{g}\left(D_{s}\right)}^{f}}\left((V, U),\left(V_{s}, 0\right)\right) \cong \operatorname{Inj}\left(V / U, V_{s}\right),
$$

where the right hand side is the set of injective linear maps. Hence we have that $I_{\left(V_{s}, 0\right)}(V, U) \cong \mathbb{F}^{\operatorname{Inj}\left(V / U, V_{s}\right)}$.

The functors $T_{(V, \varphi)}^{0}$ of Definition 2.2.1 are constructed using the splitting associated to the canonical idempotents of the finite-dimensional Boolean algebra $T_{V}^{0} K$, which gives an isomorphism for $M \in \mathrm{Ob} K-\mathcal{U}$, namely

$$
T_{V}^{0} M \cong \bigoplus_{\varphi \in \mathfrak{g}(K)(V)} T_{(V, \varphi)}^{0} M .
$$

This corresponds to a functor defined in the general framework which was introduced in Notation 5.2.1 (see [2, Chapitre 3]). Namely the forgetful functor $\mathscr{V}_{/ \mathfrak{X}}^{f} \rightarrow \mathscr{V} f$ induces a functor $\iota^{\mathfrak{X}}: \mathscr{F} \rightarrow \mathscr{F}^{\mathfrak{X}}$ by precomposition, given explicitly by $\iota^{\mathfrak{X}} F(V, x)=F(V)$. This admits a right adjoint $\Omega^{\mathfrak{X}}$ given by $\Omega^{\mathfrak{X}} G(V)=\bigoplus_{x \in \mathfrak{X}(V)} G(V, x)$.

The importance of this adjunction is through the following.

Proposition 5.2.4 [2, Proposition 3.3.10] For $\mathfrak{X}$ a presheaf of finite sets on $\mathscr{V}^{f}$, the adjunction $\iota^{\mathfrak{X}} \dashv \Omega^{\mathfrak{X}}$ induces an equivalence between $\mathscr{F}^{\mathfrak{X}}$ and the category $\mathbb{F}^{\mathfrak{X}}-\mathscr{F}^{\text {of }}$ $\mathbb{F}^{\mathfrak{X}}$-modules in $\mathscr{F}$.

There is a relative version of the above construction [2, Définition et Proposition 3.3.4]. For $\alpha: \mathfrak{X} \rightarrow \mathfrak{Y}$ a morphism of presheaves of finite sets, $\mathscr{V}_{/ \alpha}^{f}$ induces a functor 
$\alpha^{!}: \mathscr{F}^{\mathfrak{Y}} \rightarrow \mathscr{F}^{\mathfrak{X}}$ by precomposition, which admits a right adjoint $\alpha !: \mathscr{F}^{\mathfrak{X}} \rightarrow \mathscr{F}^{\mathfrak{Y}}$ given on $F \in \mathrm{Ob} \mathscr{F}^{\mathfrak{X}}$ by

$$
\left(\alpha_{!} F\right)(V, y)=\bigoplus_{x \in \alpha_{V}^{-1} y} F(V, x) .
$$

Consult [2, Chapitre 3] for the general properties.

Remark 5.2.5 For $\mathfrak{X}$ a presheaf of finite sets, $\iota^{\mathfrak{X}}=(\mathfrak{X} \rightarrow *)^{!}$and $\Omega^{\mathfrak{X}}=(\mathfrak{X} \rightarrow *)$, where $*$ is the terminal presheaf.

Example 5.2.6 For $m: K \rightarrow L$ a morphism of Noetherian unstable algebras, we have that $\mathfrak{g}(m): \mathfrak{g}(L) \rightarrow \mathfrak{g}(K)$ induces an adjunction

$$
\mathfrak{g}(m)^{!}: \mathscr{F}^{\mathfrak{g}(K)} \rightleftarrows \mathscr{F}^{\mathfrak{g}(L)}: \mathfrak{g}(m) !
$$

This is related to the induction-restriction adjunction $L \otimes_{K}-: K-U \rightleftarrows L-U$ : Restrict $_{K}^{L}$ in Theorem 5.2.8.

Definition 5.2.7 A functor $G$ of $\mathscr{F}^{\mathfrak{X}}$ is analytic if $\Omega^{\mathfrak{X}} G \in \mathrm{Ob} \mathscr{F}$ is analytic; the full subcategory of analytic functors in $\mathscr{F}^{\mathfrak{X}}$ is denoted $\mathscr{F}_{\omega}^{\mathfrak{X}}$.

Theorem 5.2.8 For $K$ a Noetherian unstable algebra, the adjunction $l: \cup \rightleftarrows \mathscr{F}: r$ induces an adjunction

$$
\mathfrak{l}_{K}: K-\mathfrak{u} \rightleftarrows \mathscr{F}^{\mathfrak{g}}(K): \mathfrak{r}_{K},
$$

where $\left(\mathfrak{l}_{K} M\right)(V, \varphi)=T_{(V, \varphi)}^{0} M$ and the underlying functor $\mathfrak{r}_{K}: \mathscr{F}^{\mathfrak{g}(K)} \rightarrow \mathcal{U}$ is the composite $r \Omega^{\mathfrak{g}(K)}$.

The functor $\mathfrak{l}_{K}$ is exact and commutes with tensor products. Moreover, $\mathfrak{l}_{K}$ induces an equivalence of categories

$$
K-U / \mathcal{N} i l \stackrel{\cong}{\rightarrow} \mathscr{F}_{\omega}^{\mathfrak{g}}(K) \text {. }
$$

For $m: K \rightarrow L$ a morphism of Noetherian unstable algebras,

(1) $\mathfrak{l}_{L}\left(L \otimes_{K}-\right): K-\mathfrak{U} \rightarrow \mathscr{F}^{\mathfrak{g}(L)}$ is naturally equivalent to $\mathfrak{g}(m) \mathfrak{l} \mathfrak{l}_{K}$;

(2) $\operatorname{Restrict}_{K}^{L} \mathfrak{r}_{L}: \mathscr{F}_{\mathfrak{g}}(L) \rightarrow K-\mathfrak{U}$ is naturally equivalent to $\mathfrak{r}_{K} \mathfrak{g}(m)$ !

Proof The functor $l: U \rightarrow \mathscr{F}$ commutes with tensor products, hence induces a functor $l: K-U \rightarrow \mathbb{F}^{\mathfrak{g}(K)}-\mathscr{F}$. The category $\mathbb{F}^{\mathfrak{g}(K)}-\mathscr{F}_{F}$ is equivalent to $\mathscr{F}^{\mathfrak{g}(K)}$ by Proposition 5.2.4, and this yields the functor $\mathfrak{l}_{K}$. Likewise, the composite $r \Omega^{\mathfrak{g}(K)}$ induces a functor to $r l(K)-\mathcal{U}$; restriction along the adjunction unit $K \rightarrow r l(K)$, which is a morphism of unstable algebras, gives $\mathfrak{r}_{K}$. That these functors are adjoint is formal and the basic properties follow from the general theory of nillocalization [8]. 
Consider the morphism $m: K \rightarrow L$. Statement (1) can be verified directly by using the explicit form of $\mathfrak{l}_{K}$ and $\mathfrak{l}_{L}$, as follows. Consider $M \in \mathrm{Ob} K-\mathfrak{U}$ and an element $\psi \in \mathfrak{g}(L)(V)$; there are natural isomorphisms

$$
T_{(V, \psi)}^{0}\left(L \otimes_{K} M\right) \cong \mathbb{F} \otimes_{T_{V}^{0} L}\left(T_{V}^{0} L \otimes_{T_{V}^{0} K} T_{V}^{0} M\right) \cong \mathbb{F} \otimes_{T_{V}^{0} K} T_{V}^{0} M,
$$

where the latter tensor product is formed with respect to $\mathfrak{g}(m) \psi \in \mathfrak{g}(K)(V)$. This establishes the first identification. Statement (2) follows by adjunction from (1).

Example 5.2.9 For $s \in \mathbb{N}$,

(1) $D_{s}-\mathcal{U} / \mathcal{N} i l$ is equivalent to the category $\mathscr{F}_{\omega}^{\mathfrak{g}\left(D_{s}\right)}$, embedded as a full subcategory of $\mathscr{F}_{\mathfrak{g}}\left(D_{s}\right)$;

(2) $H^{*} V_{s}-U / \mathcal{N} i l$ is equivalent to $\mathscr{F}_{\omega}^{\mathfrak{g}\left(H^{*} V_{s}\right)}$.

\section{Nillocalization of unstable modules over the Dickson alge- bras}

The results of Section 5.2 are applied to the categories $D_{s}-U$ to obtain the analogues of the structures considered in Section 3. The reader is referred to [2] for further results; in particular, the adjunctions considered here fit into recollement diagrams of abelian categories.

Throughout the section, the identification of $\mathscr{V}_{/ \mathfrak{g}\left(D_{s}\right)}^{f}$ given in Example 5.2.2 is used without further comment.

\subsection{Restriction}

For $0<s \in \mathbb{Z}$, the surjection $D_{s} \rightarrow \Phi D_{s-1}$ of Lemma 2.3.1 induces an inclusion $\mathfrak{g}\left(D_{s-1}\right) \cong \mathfrak{g}\left(\Phi D_{s-1}\right) \hookrightarrow \mathfrak{g}\left(D_{s}\right)$. As in Section 3.1 in the setting of modules over the Dickson algebras, there are associated adjunctions.

Proposition 6.1.1 For $1 \leq s \in \mathbb{Z}$, there is an adjunction

$$
\mathcal{R}_{s}: \mathscr{F}^{\mathfrak{g}}\left(D_{s}\right) \rightleftarrows \mathscr{F}^{\mathfrak{g}}\left(D_{s-1}\right): \mathcal{P}_{s},
$$

in which $\mathcal{R}_{s}=\mathfrak{g}\left(D_{s} \rightarrow \Phi D_{s-1}\right)$ is restriction and $\mathcal{P}_{s}=\mathfrak{g}\left(D_{s} \rightarrow \Phi D_{s-1}\right)$ ! is extension by zero.

Moreover, there are natural equivalences of exact functors

(1) $\mathcal{R}_{s} \mathfrak{l}_{D_{s}} \cong \mathfrak{l}_{D_{s-1}}\left((-) / \omega_{s}\right): D_{s}-\mathcal{U} \rightarrow \mathscr{F}_{\mathfrak{g}} \mathfrak{g}\left(D_{s-1}\right)$;

(2) $\mathcal{P}_{s} \mathfrak{l}_{D_{s-1}} \cong \mathfrak{l}_{D_{s}} \circ \operatorname{Restrict}_{s}: \Phi D_{s-1}-\mathcal{U} \rightarrow \mathscr{F}^{\mathfrak{g}}\left(D_{s}\right)$. 
Proof The identification of the functors is straightforward (cf the general results of [2, Appendice C.6]); the final statement follows from Theorem 5.2.8.

\subsection{Full restriction}

The augmentation $D_{s} \rightarrow \mathbb{F}$ gives rise to an adjunction, as in Proposition 6.1.1. In the following statement, $F \in \mathrm{Ob} \mathscr{F}$ and $G \in \mathrm{Ob} \mathscr{F}_{\mathfrak{g}}\left(D_{s}\right)$.

Proposition 6.2.1 For $s \in \mathbb{N}$, there is an adjunction

$$
\mathcal{R}_{s}^{0}: \mathscr{F}^{\mathfrak{g}}\left(D_{s}\right) \rightleftarrows \mathscr{F}_{\mathfrak{f}}: \mathcal{P}_{s}^{0},
$$

where $\mathcal{R}_{s}^{0}=\mathfrak{g}\left(D_{s} \rightarrow \mathbb{F}\right)^{!}$and $\mathcal{P}_{s}^{0}=\mathfrak{g}\left(D_{s} \rightarrow \mathbb{F}\right)$ !

Explicitly, $\mathcal{R}_{s}^{0}: \mathscr{F}^{\mathfrak{g}\left(D_{s}\right)} \rightarrow \mathscr{F}_{\mathcal{F}}$ is the restriction functor defined by $\mathcal{R}_{s}^{0} G(V):=G(V, V)$ and $\mathcal{P}_{s}^{0}: \mathscr{F} \rightarrow \mathscr{F}^{\mathfrak{g}}\left(D_{s}\right)$ is extension by zero $\mathcal{P}_{s}^{0} F(V, U)=0$ unless $V=U$, when $\mathcal{P}_{s}^{0} F(V, V)=F(V)$. In particular, the composite $\mathcal{R}_{s}^{0} \mathcal{P}_{S}^{0}$ is naturally equivalent to $1_{\mathscr{F}}$.

Moreover, there are natural equivalences of exact functors:

(1) $\mathcal{R}_{s}^{0} \mathfrak{l}_{D_{s}} \cong l Q_{s}: D_{s}-U \rightarrow \mathscr{F}$;

(2) $\mathcal{P}_{s}^{0} l \cong \mathfrak{l}_{D_{s}} \circ \operatorname{triv}_{s}: \mathcal{U} \rightarrow \mathscr{F}_{\mathfrak{e}}\left(D_{s}\right)$.

\subsection{Induction and restriction}

The canonical inclusion $D_{S} \stackrel{i}{\hookrightarrow} H^{*} V_{s}$ induces the functor $\mathscr{V}_{/ \mathfrak{g}\left(H^{*} V_{s}\right)}^{f} \rightarrow \mathscr{V}_{/ \mathfrak{g}\left(D_{s}\right)}^{f}$, which sends an object $f: V \rightarrow V_{s}$ of $\mathscr{V} f / V_{s}$ to $(V, \operatorname{ker} f)$. As above, one has the following.

Proposition 6.3.1 For $s \in \mathbb{N}$, there is an adjunction

$$
\operatorname{Ind}_{s}: \mathscr{F}^{\mathfrak{g}\left(D_{s}\right)} \rightleftarrows \mathscr{F}^{\mathfrak{g}}\left(H^{*} V_{s}\right): \operatorname{Res}_{s},
$$

where $\operatorname{Ind}_{s}=\mathfrak{g}\left(D_{s} \stackrel{i}{\hookrightarrow} H^{*} V_{s}\right) !$ and $\operatorname{Res}_{s}=\mathfrak{g}\left(D_{s} \stackrel{i}{\hookrightarrow} H^{*} V_{s}\right) !$. The functors $\operatorname{Ind}_{s}$ and $\operatorname{Res}_{s}$ are exact and $\operatorname{Ind}_{s}$ commutes with tensor products.

The induction functor is given explicitly by $\left(\operatorname{Ind}_{s} G\right)\left(V \stackrel{f}{\rightarrow} V_{s}\right)=G(V$, ker $f)$, for $G \in \mathrm{Ob} \mathscr{F}^{\mathrm{g}}\left(D_{s}\right)$.

Recall from Section 5.2 that there is an adjunction

$$
\iota^{\mathfrak{g}\left(D_{s}\right)}: \mathscr{F}_{\mathfrak{F}} \rightleftarrows \mathscr{F}^{\mathfrak{g}\left(D_{S}\right)}: \Omega^{\mathfrak{g}\left(D_{s}\right)} .
$$

In the following, $F \in \mathrm{Ob} \mathscr{F}, G \in \mathrm{Ob} \mathscr{F}^{\mathfrak{g}}\left(D_{s}\right)$ and $H \in \mathrm{Ob} \mathscr{F}_{\mathfrak{g}}\left(H^{*} V_{s}\right)$. Moreover, $I_{\left(V_{s}, 0\right)}$ denotes the injective cogenerator of $\mathscr{F}^{\mathfrak{g}}\left(D_{s}\right)$ introduced in Example 5.2.3. 
Proposition 6.3.2 For $s \in \mathbb{N}$,

(1) there are adjunctions

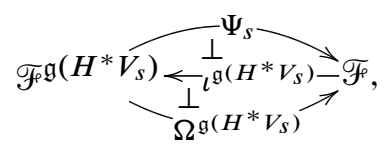

where $\Psi_{s} H(V)=H\left(V \oplus V_{s} \rightarrow V_{s}\right)$ and the functor $\Psi_{s}$ is exact and commutes with tensor products;

(2) $\operatorname{Ind}_{s} \iota^{\mathfrak{g}\left(D_{s}\right)}$ is naturally isomorphic to $\iota^{\mathfrak{g}\left(H^{*} V_{s}\right)}$ and $\Omega^{\mathfrak{g}\left(D_{s}\right)} \operatorname{Res}_{s}$ is naturally isomorphic to $\Omega^{\mathfrak{g}\left(H^{*} V_{s}\right)}$;

(3) the map $\Psi_{s} \operatorname{Ind}_{s}: \mathscr{F}^{\mathfrak{g}}\left(D_{s}\right) \rightarrow \mathscr{F}$ is determined by $\Psi_{s} \operatorname{Ind}_{s} G(V)=G\left(V \oplus V_{s}, V\right)$; it is exact, commutes with tensor products and is left adjoint to the functor $\operatorname{Res}_{s} \iota^{\mathfrak{g}\left(H^{*} V_{s}\right)}: \mathscr{F} \rightarrow \mathscr{F}^{\mathfrak{g}\left(D_{s}\right)} ;$

(4) $\operatorname{Res}_{s} l^{\mathfrak{g}\left(H^{*} V_{s}\right)}: \mathscr{F}_{\mathcal{F}}^{\rightarrow} \mathscr{F}_{\mathfrak{g}} \mathfrak{g}\left(D_{s}\right)$ is exact and is naturally equivalent to the functor

$$
F \mapsto \iota^{\mathfrak{g}\left(D_{s}\right)} F \otimes I_{\left(V_{s}, 0\right)} .
$$

Moreover, there are natural isomorphisms

$$
\begin{aligned}
l \mathrm{Fix}_{s} & \cong \Psi_{s} \mathfrak{l}_{H^{*} V_{s}}: H^{*} V_{s}-\mathcal{U} \rightarrow \mathscr{F}, \\
l \text { Fix }_{s}\left(H^{*} V_{s} \otimes_{D_{s}}-\right) & \cong \Psi_{s} \operatorname{Ind}_{s} \mathfrak{l}_{D_{s}}: D_{s}-\mathcal{U} \rightarrow \mathscr{F} .
\end{aligned}
$$

Proof The first statement follows from the natural isomorphism

$$
\operatorname{Hom}_{\mathscr{V} f} / V_{s}\left(\left(A \rightarrow V_{S}\right),\left(V \oplus V_{s} \stackrel{\mathrm{pr}}{\longrightarrow} V_{s}\right)\right) \cong \operatorname{Hom}_{\mathscr{V} f}(A, V) .
$$

The remaining numbered statements are straightforward and follow from [2, Définition et Proposition 3.3.4].

The functor $l$ Fix $_{s}$ is left adjoint to the functor

$$
F \mapsto H^{*} V_{s} \otimes r F \cong r\left(I_{V_{s}} \otimes F\right),
$$

for $F \in \mathrm{Ob} \mathscr{F}$. The latter is isomorphic to $\mathfrak{r}_{H^{*} V_{s} \iota^{\mathfrak{g}}\left(H^{*} V_{s}\right)} F$, since the underlying functor of $\mathfrak{r}_{H^{*} V_{s}}$ is $r \Omega^{\mathfrak{g}\left(H^{*} V_{s}\right)}$ and $\Omega^{\mathfrak{g}\left(H^{*} V_{s}\right)} \iota^{\mathfrak{g}\left(H^{*} V_{s}\right)} F \cong I_{V_{s}} \otimes F$ (cf [2, Définition et Proposition 3.3.4]). Since $\mathfrak{l}_{H^{*} V_{s}}$ is left adjoint to $\mathfrak{r}_{H^{*} V_{s}}$ and $\Psi_{s}$ is left adjoint to $\iota^{\mathfrak{g}\left(H^{*} V_{s}\right)}$, it follows by unicity of adjoints that $l \mathrm{Fix}_{S}$ is equivalent to $\Psi_{s} \mathfrak{l}_{H^{*}} V_{s}$.

The final identification follows from the natural isomorphism given by Theorem 5.2.8, $\mathfrak{l}_{H^{*} V_{s}}\left(H^{*} V_{s} \otimes_{D_{s}}-\right) \cong \operatorname{Ind}_{s} \mathfrak{l}_{D_{s}}$. 
Example 6.3.3 Proposition 6.3.2(4) contains the isomorphism

$$
\operatorname{Res}_{s} i^{\mathfrak{g}\left(H^{*} V_{s}\right)} \mathbb{F} \cong I_{\left(V_{s}, 0\right)} .
$$

The importance of $I_{\left(V_{s}, 0\right)}$ is shown by the isomorphism $\mathfrak{r}_{D_{s}} I_{\left(V_{s}, 0\right)} \cong \operatorname{Restrict}_{D_{s}}^{H^{*} V_{s}} H^{*} V_{s}$ in $D_{s}-U$, which follows from Theorem 5.2.8.

\section{The Singer functors up to nilpotent unstable modules}

This section introduces the functors $\kappa_{s}: \mathscr{F}_{F} \rightarrow \mathscr{F}_{\mathfrak{F}}\left(D_{s}\right)$ which model the Singer functors up to nilpotent unstable modules. The proof that these correspond to the functors $R_{S}$ is postponed until Section 8 .

\subsection{Avatars of the Singer functors}

Some of the material of this section is available in [2] under a dual formulation using comodules over Boolean coalgebras.

Definition 7.1.1 For $s \in \mathbb{N}$, let $\kappa_{s}: \mathscr{F}_{F} \rightarrow \mathscr{F}_{\mathfrak{g}}\left(D_{s}\right)$ denote the functor defined on $F \in \mathrm{Ob} \mathscr{F}$ by

$$
\left(\kappa_{s} F\right)(V, W):=F(W)
$$

Notation 7.1.2 For $s \in \mathbb{N}, V \in \mathrm{Ob}^{q} f$, let $\operatorname{Stab}\left(V, V \oplus V_{s}\right) \subset \operatorname{Aut}\left(V \oplus V_{s}\right)$ denote the pointwise stabilizer of $V$.

The following is clear.

Lemma 7.1.3 Let $V, W \in \mathrm{Ob}^{\mathscr{}} f$ and $f: V \rightarrow W$ be a linear morphism. Then

(1) $\operatorname{Stab}\left(V, V \oplus V_{S}\right)$ is isomorphic to the semidirect product $\operatorname{Hom}\left(V_{s}, V\right) \rtimes \operatorname{Aut}\left(V_{s}\right)$, where $\operatorname{Aut}\left(V_{S}\right)$ acts on the right on $\operatorname{Hom}\left(V_{s}, V\right)$ by precomposition;

(2) the action of $\operatorname{Aut}\left(V \oplus V_{S}\right)$ on $V \oplus V_{s}$ induces an action of $\operatorname{Stab}\left(V, V \oplus V_{S}\right)$ on $\left(V \oplus V_{s}, V\right) \in \mathrm{Ob}^{f}{ }_{/ \mathfrak{g}\left(D_{s}\right)}^{f}$

(3) $f: V \rightarrow W$ induces a group morphism $\operatorname{Stab}\left(V, V \oplus V_{s}\right) \rightarrow \operatorname{Stab}\left(W, W \oplus V_{s}\right)$ which, with respect to the semidirect product decomposition, is induced by $\operatorname{Hom}\left(V_{s}, f\right): \operatorname{Hom}\left(V_{s}, V\right) \rightarrow \operatorname{Hom}\left(V_{s}, W\right)$.

In the following statement, $G \in \mathrm{Ob} \mathscr{F}^{g}\left(D_{s}\right), V \in \mathrm{Ob} \mathscr{V}^{f}$ and $(V, U) \in \mathrm{Ob} \mathscr{V}_{/ \mathfrak{g}\left(D_{s}\right)}^{f}$. 
Proposition 7.1.4 Let $s$ be a natural number.

(1) The functor $\kappa_{s}: \mathscr{F}_{F} \rightarrow \mathscr{F}^{\mathfrak{g}\left(D_{s}\right)}$ is exact and commutes with tensor products, limits and colimits.

(2) There is a natural monomorphism $\kappa_{S} \hookrightarrow \iota^{\mathfrak{g}\left(D_{s}\right)}$ of functors from $\mathscr{F}_{F}$ to $\mathscr{F}^{\mathfrak{g}\left(D_{s}\right)}$.

(3) The functor $\kappa_{s}$ is left adjoint to $\mathcal{R}_{s}^{0}$ and admits a left adjoint $\mathfrak{a}_{s}: \mathscr{F}^{\mathfrak{g}}\left(D_{s}\right) \rightarrow \mathscr{F}$ given by $\mathfrak{a}_{s} G(V) \cong G\left(V \oplus V_{s}, V\right) / \operatorname{Stab}\left(V, V \oplus V_{s}\right)$.

(4) The adjunction counit $\mathfrak{a}_{s} \kappa_{s} \rightarrow 1_{\mathscr{F}}$ is an isomorphism.

(5) The adjunction unit $1_{\mathscr{F}} \rightarrow \mathcal{R}_{S}^{0} \kappa_{S}$ is an isomorphism.

Proof The first statement is clear; for the second, the inclusion $U \subset V$ induces a natural morphism $F(U) \rightarrow F(V)$.

The fact that $\mathcal{R}_{s}^{0}$ is right adjoint to $\kappa_{s}$ follows from the isomorphism

$$
\operatorname{Hom}_{\mathscr{V}_{/ \mathfrak{g}\left(D_{s}\right)}^{f}}((V, V),(A, B)) \cong \operatorname{Hom}_{\mathscr{V} f}(V, B),
$$

for $V \in \mathrm{Ob} \mathscr{V}^{f}$ and $(A, B) \in \mathrm{Ob} \mathscr{V}_{/ \mathfrak{g}\left(D_{s}\right)}^{f}$.

The left adjoint $\mathfrak{a}_{s}$ exists for formal reasons and $\mathfrak{a}_{s}$ is a right exact functor which preserves projective objects. Lemma 7.1.3 implies that the association given by $V \mapsto G\left(V \oplus V_{s}, V\right) / \operatorname{Stab}\left(V, V \oplus V_{s}\right)$ defines a right exact functor. Hence, since $\mathscr{F}_{\mathfrak{g}} \mathrm{g}\left(D_{s}\right)$ has enough projectives (see Example 5.2.3), it suffices to check that this coincides with $\mathfrak{a}_{s}$ on the full subcategory of projective objects in $\mathscr{F}_{\mathfrak{g}}\left(D_{s}\right)$. Let $(A, B)$ and $V$ be as above, then there is a natural isomorphism

$$
\operatorname{Hom}_{\mathscr{V} f}(A, B) \cong \operatorname{Hom}_{\mathscr{V}_{/ \mathfrak{g}\left(D_{s}\right)}^{f}}\left((A, B),\left(V \oplus V_{s}, V\right)\right) / \mathrm{Stab}\left(V, V \oplus V_{s}\right) .
$$

It follows that there is a natural isomorphism

$$
\mathfrak{a}_{S} P_{(A, B)}(V) \cong P_{(A, B)}\left(V \oplus V_{s}, V\right) / \operatorname{Stab}\left(V, V \oplus V_{S}\right),
$$

as required. The identification of the adjunction morphisms is clear.

Recall from Example 5.1.2 that, for $U \in \mathrm{Ob} \mathscr{V}^{f}, I_{U}$ is the injective functor $\mathbb{F}^{\operatorname{Hom}_{v} f(-, U)}$ of $\mathscr{F}$, which is contravariantly functorial in $U$.

The composite functor $\Omega^{\mathfrak{g}\left(D_{S}\right)} \kappa_{S}: \mathscr{F}_{F} \rightarrow \mathscr{F}_{F}$ is of particular interest; the following result is required to relate the functor $\kappa_{S}$ to the Singer functor $R_{S}$.

Proposition 7.1.5 For $U \in \mathrm{Ob}^{f} f$, there is a natural isomorphism

$$
\Omega^{\mathfrak{g}\left(D_{s}\right)} \kappa_{S} I_{U} \cong I_{U \oplus V_{s}}^{\mathrm{Stab}\left(U, U \oplus V_{s}\right)} .
$$


Proof The functor $\Omega^{\mathfrak{g}\left(D_{s}\right)} \kappa_{S}$ is right adjoint to the functor $\mathfrak{a}_{s} \iota^{\mathfrak{g}\left(D_{s}\right)}$, which identifies with the composite functor $F \mapsto\left(\Delta_{V_{s}} F\right) / \operatorname{Stab}\left(-,-\oplus V_{s}\right)$, by Proposition 7.1.4. The functor $\Delta_{V_{s}}$ is left adjoint to the functor $-\otimes I_{V_{s}}$ (see Example 5.1.2), hence it follows that there is a natural isomorphism

$$
\operatorname{Hom}_{\mathscr{F}}\left(\left(\Delta_{V_{s}} F\right) / \operatorname{Stab}\left(-,-\oplus V_{S}\right), I_{U}\right) \cong \operatorname{Hom}_{\mathscr{F}}\left(F,\left(I_{U} \otimes I_{V_{s}}\right)^{\operatorname{Stab}\left(U, U \oplus V_{s}\right)}\right)
$$

and the group action is induced by the natural right action on $I_{U} \otimes I_{V_{s}} \cong I_{U \oplus V_{s}}$. The result follows.

We record the following, which is clear.

Lemma 7.1.6 The composite functor $\Psi_{s} \operatorname{Ind}_{s} \kappa_{s}$, considered as a functor $\mathscr{F}_{\mathscr{F}} \rightarrow \mathscr{F}_{F}$, is naturally equivalent to the identity functor.

\subsection{Copresentations of $\kappa_{s}$}

In order to understand the underlying object of $\Omega^{\mathfrak{g}\left(D_{s}\right)} \kappa_{s}$, an alternative description is used; this is obtained by giving a copresentation of $\kappa_{S}$ via an equalizer diagram.

Recall that a diagram

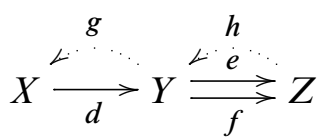

is a split equalizer if there exist morphisms $g: Y \rightarrow X$ and $h: Z \rightarrow Y$ such that $g d=1_{X}, h f=1_{Y}$ and $d g=h e: Y \rightarrow Y$. A split equalizer is, in particular, an equalizer diagram.

Notation 7.2.1 For $s \in \mathbb{N}$, let $\delta_{s}: \mathscr{V}_{/ \mathfrak{g}\left(D_{s}\right)}^{f} \rightarrow \mathscr{V}^{f}$ denote the functor $(V, U) \mapsto V \oplus V / U$.

Lemma 7.2.2 For $(V, U) \in \mathrm{Ob}^{\mathscr{V}} / \mathfrak{g}\left(D_{s}\right)$, there is a natural equalizer diagram in $\mathscr{V} f$

$$
U \longrightarrow V \underset{1 \prod q}{\stackrel{1 \prod 0}{\longrightarrow}} \oplus V / U=\delta_{S}(V, U),
$$

where $q: V \rightarrow V / U$ is the quotient morphism. If $s: V / U \rightarrow V$ is a section of $q$, then the equalizer is split by the morphisms $1 \bigsqcup-s: V \oplus V / U \rightarrow V$ and the induced projection $V \rightarrow U$.

In the following, the notation introduced in Example 5.2.3 for the injective cogenerators of $\mathscr{F F}^{\mathfrak{g}}\left(D_{s}\right)$ is used. 
Proposition 7.2.3 For $s \in \mathbb{N}$,

(1) there is a monomorphism

$$
\left(\delta_{S}\right)^{!}(-) \hookrightarrow I_{\left(V_{s}, 0\right)} \otimes \iota^{\mathfrak{g}\left(D_{s}\right)} \Delta_{V_{s}}(-)
$$

of functors from $\mathscr{F}$ to $\mathscr{F} \mathfrak{g}\left(D_{s}\right)$;

(2) the equalizer of Lemma 7.2.2 induces an equalizer diagram of functors from $\mathscr{F}$ to $\mathscr{F}_{\mathfrak{g}}\left(D_{s}\right)$

$$
\kappa_{S} \longrightarrow l^{\mathfrak{g}}\left(D_{s}\right) \longrightarrow\left(\delta_{S}\right) !
$$

and hence an equalizer diagram

$$
\kappa_{S} \longrightarrow \iota^{\mathfrak{g}\left(D_{s}\right)} \longrightarrow I_{\left(V_{s}, 0\right)} \otimes \iota^{\mathfrak{g}\left(D_{s}\right)} \Delta_{V_{s}}(-) ;
$$

(3) applying the functor $\Omega^{\mathfrak{g}\left(D_{s}\right)}$ to the equalizer (1) gives the equalizer diagram of functors from $\mathscr{F}_{F}$ to $\mathbb{F}^{\mathfrak{g}^{\left(D_{s}\right)}-\mathscr{F}_{\mathrm{F}}}$

$$
\Omega^{\mathfrak{g}\left(D_{s}\right)} \kappa_{s} \longrightarrow \mathbb{F}^{\mathfrak{g}\left(D_{s}\right)} \otimes(-) \underset{\tau}{\stackrel{\sigma}{\longrightarrow}} I_{V_{s}} \otimes \Delta_{V_{s}}(-),
$$

where $\sigma, \tau$ are induced by the natural morphisms of $\mathscr{F}$

$$
F \underset{\tau_{F}^{\prime}}{\stackrel{\sigma_{F}^{\prime}}{\longrightarrow}} I_{V_{s}} \otimes \Delta_{V_{s}} F,
$$

where $\sigma_{F}^{\prime}$ is the tensor product of the unit $\mathbb{F} \rightarrow I_{V_{s}}$ with the natural inclusion $F \cong \Delta_{0} F \hookrightarrow \Delta_{V_{s}} F$ and $\tau_{F}^{\prime}$ is the adjunction unit for $\Delta_{V_{s}} \dashv\left(I_{V_{s}} \otimes-\right)$.

Proof The first statement can be established by an adjunction argument or be seen as follows. Recall from Example 5.2.3 there is an identification $I_{\left(V_{s}, 0\right)}(V, U) \cong \mathbb{F}^{\operatorname{Inj}\left(V / U, V_{s}\right)}$. Consider $F \in \mathrm{Ob} \mathscr{F}$; the natural monomorphism

$$
\left(\delta_{s}\right)^{!} F(V, U) \rightarrow I_{\left(V_{s}, 0\right)}(V, U) \otimes \iota^{\mathfrak{g}\left(D_{s}\right)} \Delta_{V_{s}} F(V, U) \cong \mathbb{F}^{\operatorname{Inj}\left(V / U, V_{s}\right)} \otimes F\left(V \oplus V_{s}\right)
$$

has component indexed by a monomorphism $V / U \hookrightarrow V_{s}$ given by the induced morphism $F(V \oplus V / U) \rightarrow F\left(V \oplus V_{s}\right)$.

The first diagram of the second statement is obtained by precomposition with the natural diagram of Lemma 7.2.2. Since limits are computed in $\mathscr{F}^{\mathfrak{g}}\left(D_{s}\right)$ pointwise, it suffices to show that, for $F \in \mathrm{Ob} \mathscr{F}$ and $(V, U) \in \mathrm{Ob}{ }_{/ \mathfrak{g}\left(D_{s}\right)}^{f}$, the diagram in $\mathscr{V}$

$$
F(U) \rightarrow F(V) \longrightarrow F(V \oplus V / U)
$$


is a split equalizer; this follows from Lemma 7.2.2, since split equalizers are preserved by functors. Composing with the monomorphism of the first statement gives the second equalizer diagram.

The third statement follows by applying the exact functor $\Omega^{\mathfrak{g}\left(D_{s}\right)}$ to the previous equalizer diagram, using [2, Définition et Proposition 3.3.4] to identify the functors. Namely, for $F \in \mathrm{Ob} \mathscr{F}$, there is a natural isomorphism $\Omega^{\mathfrak{g}\left(D_{s}\right)} \iota^{\mathfrak{g}\left(D_{s}\right)} F \cong \mathbb{F}^{\mathfrak{g}\left(D_{s}\right)} \otimes F$ in $\mathbb{F}^{\mathfrak{g}\left(D_{s}\right)}$ - $\mathscr{F}$ and there are natural isomorphisms $\Omega^{\mathfrak{g}\left(D_{s}\right)}\left(I_{\left(V_{s}, 0\right)} \otimes \iota^{\mathfrak{g}\left(D_{s}\right)} \Delta_{V_{s}} F\right) \cong$ $\left(\Omega^{\mathfrak{g}\left(D_{s}\right)} I_{\left(V_{s}, 0\right)}\right) \otimes \Delta_{V_{s}} F \cong I_{V_{s}} \otimes \Delta_{V_{s}} F$, where the second isomorphism follows from $\Omega^{\mathfrak{g}\left(D_{s}\right)} I_{\left(V_{s}, 0\right)} \cong I_{V_{s}}$, which is a formal consequence of the adjunction $\iota^{\mathfrak{g}\left(D_{s}\right)} \dashv \Omega^{\mathfrak{g}\left(D_{s}\right)}$.

The identification of the natural transformations $\sigma, \tau$ follows by unravelling the definitions.

Remark 7.2.4 Lemma 7.1.6 shows that $\Psi_{s} \operatorname{Ind}_{s} \kappa_{s}$ is naturally equivalent to the identity functor. It is instructive to see how this can be recovered from the copresentation of $\kappa_{S}$ given in Proposition 7.2.3; this is a guiding principle in the proof of Theorem 8.2.2.

The functor $\Psi_{s} \operatorname{Ind}_{s}$ is exact and an explicit description is given in Proposition 6.3.2. Applying $\Psi_{s} \mathrm{Ind}_{s}$ to the parallel arrows of (1), evaluated on $F \in \mathrm{Ob} \mathscr{F}$, gives the diagram

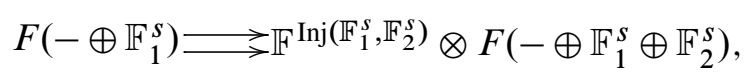

where the suffixes are used to distinguish the direct factors. Fixing an element $\alpha \in \operatorname{Inj}\left(\mathbb{F}_{1}^{s}, \mathbb{F}_{2}^{s}\right) \cong \operatorname{Aut}\left(\mathbb{F}^{s}\right)$, the associated components evaluated on $V \in \mathrm{Ob}^{f}$ are

$$
F\left(V \oplus \mathbb{F}_{1}^{s}\right) \underset{F\left(1_{V} \prod 1_{\mathbb{F}} \| \alpha\right)}{\stackrel{F\left(1_{V} \prod 1_{\mathbb{F}} s \prod 0\right)}{\longrightarrow}} F\left(V \oplus \mathbb{F}_{1}^{s} \oplus \mathbb{F}_{2}^{s}\right)
$$

It is clear that

(1) the natural inclusion $F(V) \hookrightarrow F\left(V \oplus \mathbb{F}_{1}^{s}\right)$ equalizes the parallel arrows,

(2) for $\alpha=1_{\mathbb{F}^{s}}$, the equalizer of (3) is $F(V)$,

where the second point is seen by applying Lemma 7.2.2 to $\left(V \oplus \mathbb{F}_{1}^{s} \oplus \mathbb{F}_{2}^{s}, V \oplus \mathbb{F}_{1}^{s}\right)$.

It follows formally that there is a natural isomorphism $\Psi_{s} \operatorname{Ind}_{s} \kappa_{s} F(V) \cong F(V)$, as expected. 


\subsection{Compatibility}

The functors $\kappa_{S}$ introduced above are related under restriction and induction, via the adjunctions $\mathcal{R}_{S}: \mathscr{F}^{g}\left(D_{s}\right) \rightleftarrows \mathscr{F}^{\mathfrak{g}}\left(D_{s-1}\right): \mathcal{P}_{S}$ of Proposition 6.1.1.

Proposition 7.3.1 Let $s$ be a positive integer.

(1) There is a natural isomorphism $\mathcal{R}_{s} \iota^{\mathfrak{g}\left(D_{s}\right)} \rightarrow \iota^{\mathfrak{g}\left(D_{s-1}\right)}$ and the adjoint $\iota^{\mathfrak{g}\left(D_{s}\right)} \rightarrow$ $\mathcal{P}_{s} \iota^{\mathfrak{g}\left(D_{s-1}\right)}$ is a surjection with kernel $\mathcal{R}^{s} \mathcal{P}^{s} \iota^{\mathfrak{g}\left(D_{s}\right)}$.

(2) There is a natural isomorphism $\mathcal{R}_{s} \kappa_{s} \rightarrow \kappa_{s-1}$ and the adjoint $\kappa_{s} \rightarrow \mathcal{P}_{s} \kappa_{s-1}$ is a surjection with kernel $\mathcal{R}^{s} \mathcal{P}^{s} \kappa_{s}$.

(3) There is a commutative diagram of natural transformations

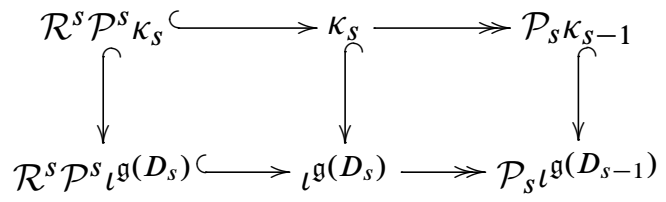

in which the rows are short exact sequences.

Proof Straightforward.

Remark 7.3.2 For $F \in \mathrm{Ob} \mathscr{F}$, the short exact sequence $\mathcal{R}^{s} \mathcal{P}^{s} \kappa_{S} F \rightarrow \kappa_{S} F \rightarrow \mathcal{P}_{s} \kappa_{s-1} F$ is the analogue of the short exact sequence $\omega_{s} R_{S} M \rightarrow R_{S} M \rightarrow \Phi R_{s-1} M$ from Proposition 4.1.3.

\section{Deeper properties of the Singer functors}

This section introduces an approximation $\widetilde{R}_{S}$ to the functor $R_{S}$, by lifting the copresentation of $\kappa_{S}$ of Section 7.2 to the category $D_{S}-U$. The functors $\widetilde{R}_{S}$ and $R_{S}$ are shown to coincide on reduced unstable modules; in general, $\widetilde{R}_{S}$ is the $\omega_{S}$-closure of $R_{S}$.

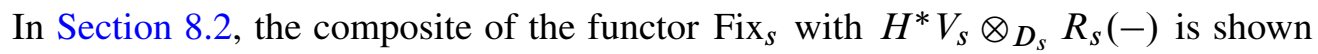
to be naturally equivalent to the identity. This is used to deduce that the functor $R_{S}$ defines a fully-faithful embedding of $\mathcal{U}$ in $D_{s}-U$. 


\subsection{Lifting the functor $\Omega^{\mathfrak{g}\left(D_{s}\right)} \kappa_{s}$ to $U$}

The parallel arrows of diagram (2) of Proposition 7.2.3 lift to a natural diagram in $D_{s}-U$ :

$$
D_{s} \otimes M \underset{\tau_{M}}{\stackrel{\sigma_{M}}{\longrightarrow}} H^{*} V_{s} \otimes T_{V_{s}} M
$$

for $M \in \mathrm{Ob} u$, where

(1) $\sigma_{M}$ is the tensor product of the inclusions $M \cong T_{0} M \hookrightarrow T_{V_{s}} M$ and $D_{S} \hookrightarrow H^{*} V_{s}$;

(2) $\tau_{M}$ is the morphism of $D_{s}$-modules induced by the adjunction unit (in $U$ ) $M \rightarrow H^{*} V_{s} \otimes T_{V_{s}} M$.

Remark 8.1.1 The context should ensure that there is no ambiguity with the notation used in Section 7.2.

Definition 8.1.2 For $s \in \mathbb{N}$, let $\widetilde{R}_{s}: \mathcal{U} \rightarrow D_{s}-\mathcal{U}$ be the functor determined on an unstable module $M$ by

$$
\widetilde{R}_{S} M:=\operatorname{ker}\left\{D_{s} \otimes M \underset{\tau_{M}}{\stackrel{\sigma_{M}}{\longrightarrow}} H^{*} V_{s} \otimes T_{V_{s}} M\right\} .
$$

Recall that an unstable module $N$ is locally finite if and only if the natural monomorphism $N \hookrightarrow T N$ is an isomorphism [19, Theorem 6.2.1].

Proposition 8.1.3 For $s \in \mathbb{N}$,

(1) there is a natural monomorphism $\widetilde{R}_{S} \hookrightarrow D_{s} \otimes-$;

(2) $\widetilde{R}_{s}: U \rightarrow D_{s}-U$ is left exact and commutes with coproducts;

(3) $\widetilde{R}_{s}$, considered as a functor with values in $U$, preserves the class of reduced (respectively nilclosed) unstable modules;

(4) $\widetilde{R}_{S}$ takes values in the class of $\omega_{s}$-closed objects of $D_{s}-U$;

(5) for $M, X$ unstable modules, with $X$ locally finite, there is a natural isomorphism $\widetilde{R}_{S}(M \otimes X) \cong\left(\widetilde{R}_{S} M\right) \otimes X$; in particular, $\widetilde{R}_{S}$ commutes with suspension.

Proof The first three statements are straightforward. The fact that $\widetilde{R}_{S}$ takes values in the category of $\omega_{s}$-closed unstable $D_{s}$-modules follows from Proposition 3.1.4.

For the final statement, since $X$ is locally finite, the natural inclusion $X \hookrightarrow T_{V_{S}} X$ is an isomorphism, hence there is a natural isomorphism $T_{V_{s}}(M \otimes X) \cong\left(T_{V_{s}} M\right) \otimes X$. It is straightforward to check that, via this isomorphism, there are identifications $\sigma_{M \otimes X}=\sigma_{M} \otimes 1_{X}$ and $\tau_{M \otimes X}=\tau_{M} \otimes 1_{X}$, which implies the result. 
Proposition 8.1.4 For $s \in \mathbb{N}$, the restrictions of the functors $R_{S}$ and $\widetilde{R}_{S}$ to the full subcategory of nilclosed unstable modules are naturally isomorphic.

Proof By Proposition 4.1.3, $R_{s}$ commutes with coproducts, preserves the class of nilpotent unstable modules and sends nilclosed unstable modules to nilclosed unstable modules; Proposition 8.1.3 establishes the analogous properties for $\widetilde{R}_{S}$. It follows from Lemma A.2.2 that it is sufficient to show that the two functors coincide on the full subcategory of $U$ with objects $\left\{H^{*} V_{s} \mid s \in \mathbb{N}\right\}$. Since there are natural monomorphisms $R_{S} \hookrightarrow D_{S} \otimes-$ and $\widetilde{R}_{S} \hookrightarrow D_{S} \otimes-$, by composing with the natural monomorphism $i \otimes-: D_{s} \otimes-\hookrightarrow H^{*} V_{s} \otimes-$, it is sufficient to show that the images of $R_{s} H^{*} V$ and $\widetilde{R}_{S} H^{*} V$ in $H^{*} V_{s} \otimes H^{*} V$ coincide, for every $V \in \mathrm{Ob}^{\mathscr{}} f$.

Lannes and Zarati prove that $R_{S} H^{*} V$ is isomorphic to $H^{*}\left(V \oplus V_{s}\right)^{\operatorname{Stab}\left(V, V \oplus V_{s}\right)}$ in [11, Section 5.4.7.5]; Proposition 7.1.5 implies that this is isomorphic to $\widetilde{R}_{S} H^{*} V$. The result follows.

Theorem 8.1.5 For $s \in \mathbb{N}$, there is a natural monomorphism

$$
\gamma_{s}: R_{S} \hookrightarrow \widetilde{R}_{S}
$$

of functors $U \rightarrow D_{s}-U$ such that

(1) $\gamma_{s}$ identifies $\widetilde{R}_{S}$ as the $\omega_{s}$-closure of $R_{S}$;

(2) the morphism $\gamma_{s}: R_{S} M \rightarrow \widetilde{R}_{S} M$ is an isomorphism if $M$ is reduced.

Proof The construction of the natural monomorphism $\gamma_{s}$ generalizes the argument employed in the proof of Lemma A.2.2. Recall (cf [19, Section 3.11]) that the set of objects $H^{*} V_{m} \otimes J(n)$ (where $J(n)$ denotes the $n$-th Brown-Gitler module), indexed over nonnegative integers $m, n$, forms a set of injective cogenerators of $u$ and that, since $U$ is locally Noetherian, any unstable module $M$ admits a copresentation of the form $0 \rightarrow M \rightarrow I^{0} \rightarrow I^{1}$, where each $I^{j}$ is a coproduct of objects of this form. Hence, writing $W$ for $V_{m}$, it suffices to show that there is a factorization

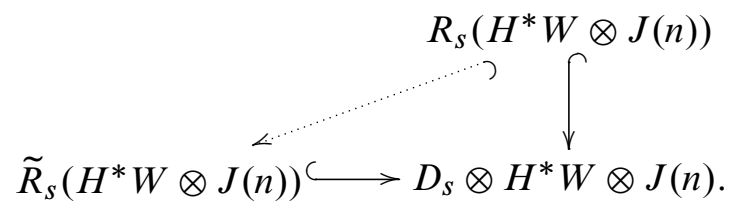

Now we have that $R_{S}\left(H^{*} W \otimes J(n)\right) \cong R_{s} H^{*} W \otimes D_{s} R_{S} J(n), D_{s} \otimes H^{*} W \otimes J(n) \cong$ $\left(D_{s} \otimes H^{*} W\right) \otimes_{D_{s}}\left(D_{s} \otimes J(n)\right)$ and the vertical inclusion is the tensor product over $D_{s}$ of $R_{S} H^{*} W \hookrightarrow D_{s} \otimes H^{*} W$ and $R_{S} J(n) \hookrightarrow D_{s} \otimes J(n)$; the latter is the $\omega_{s}$-closure of $R_{S} J(n)$, by Proposition 4.1.5. 
Similarly, by Proposition 8.1.3, the horizontal inclusion identifies with the tensor product over $D_{s}$ of $\widetilde{R}_{s} H^{*} W \hookrightarrow D_{s} \otimes H^{*} W$ and the isomorphism $\widetilde{R}_{s} J(n) \rightarrow D_{s} \otimes J(n)$. Since the images of $R_{s} H^{*} W$ and $\widetilde{R}_{s} H^{*} W$ in $D_{s} \otimes H^{*} W$ coincide, by Proposition 8.1.3, this provides the required factorization.

The cokernel of $R_{S}\left(H^{*} W \otimes J(n)\right) \hookrightarrow \widetilde{R}_{S}\left(H^{*} W \otimes J(n)\right)$ is $\omega_{s}$-torsion, by the above discussion. It follows that the cokernel of $R_{S} M \rightarrow \widetilde{R}_{S} M$ is $\omega_{s}$-torsion, for any unstable module $M$; since, $\widetilde{R}_{S} M$ is $\omega_{s}$-closed (by Proposition 8.1.3), this exhibits $\widetilde{R}_{S} M$ as the $\omega_{s}$-closure of $R_{S} M$.

To prove the final statement, one can forget the $D_{s}-$ module structure. The result follows from Lemma A.3.1, since $\gamma_{s}$ is an isomorphism on nilclosed unstable modules, by Proposition 8.1.4.

Corollary 8.1.6 For $s \in \mathbb{N}$, there is a natural isomorphism $\mathfrak{l}_{D_{s}} R_{s} \cong \kappa_{s} l$ of functors from $U$ to $\mathscr{F}_{\mathfrak{g}}\left(D_{s}\right)$.

Proof The functors $\mathfrak{l}_{D_{s}} R_{S}$ and $\kappa_{S} l$ are exact and send nilpotent unstable modules to zero. Hence, by Lemma A.2.1, it suffices to prove that the two functors coincide naturally on the full subcategory of nilclosed unstable modules. On this subcategory, $\gamma_{s}: R_{S} \hookrightarrow \widetilde{R}_{s}$ is a natural isomorphism, by Theorem 8.1 .5 , hence it suffices to prove that there is a natural isomorphism $\mathfrak{l}_{D_{S}} \widetilde{R}_{S} \cong \kappa_{S} l$. This is by construction: applying the functor $\mathfrak{l}_{D_{s}}$ to the equalizer diagram defining $\widetilde{R}_{S}$ gives the copresentation of $\Omega^{\mathfrak{g}\left(D_{s}\right)} \kappa_{S}$ given in Proposition 7.2.3.

\subsection{The composite of $R_{s}$ and $\operatorname{Fix}_{s}\left(H^{*} V_{s} \otimes_{D_{s}}-\right)$}

Under the correspondence between $D_{s}-\mathcal{U} / \mathcal{N} i l$ and the category $\mathscr{F}_{\mathfrak{g}}\left(D_{s}\right)$ given by Theorem 5.2.8, the Singer functor corresponds to the functor $\kappa_{S}$ (by Corollary 8.1.6) and the functor $\Psi_{s} \operatorname{Ind}_{s}$ corresponds to the functor Fix ${ }_{s}\left(H^{*} V_{s} \otimes_{D_{s}}-\right)$ (by Proposition 6.3.2). Lemma 7.1.6 states that the composite $\Psi_{s} \operatorname{Ind}_{s} \kappa_{S}$ is isomorphic to the identity functor; the purpose of this section is to establish the corresponding result at the level of unstable modules.

Recall that $i: D_{S} \hookrightarrow H^{*} V_{s}$ denotes the canonical inclusion and that Proposition 2.3.2 implies that $T_{\left(V_{s}, i\right)}$ induces a functor $D_{s}-\mathcal{U} \rightarrow H^{*} V_{s}-\mathcal{U}$. The following result is the key input.

Lemma 8.2.1 For $s \in \mathbb{N}$ and $M \in \mathrm{Ob} D_{s}-u$,

$$
\operatorname{Fix}_{S}\left(H^{*} V_{s} \otimes_{D_{s}} M\right) \cong \mathbb{F} \otimes_{H^{*} V_{s}} T_{\left(V_{s}, i\right)} M .
$$

In particular, there is an isomorphism of unstable algebras

$$
\operatorname{Fix}_{s}\left(H^{*} V_{s} \otimes_{D_{s}} H^{*} V_{s}\right) \cong \mathbb{F}^{\mathrm{Aut} V_{s}} .
$$


Proof By [10, 4.4.3], $\operatorname{Fix}_{s} X \cong \mathbb{F} \otimes_{T_{V_{s}} H^{*} V_{s}} T_{V_{s}} X$, for $X \in \mathrm{Ob} H^{*} V_{s}-\mathcal{U}$. The $T$-functor commutes with tensor products, so, taking $X=H^{*} V_{s} \otimes_{D_{s}} M$, there are natural isomorphisms

$$
\begin{aligned}
\operatorname{Fix}_{s}\left(H^{*} V_{s} \otimes_{D_{s}} M\right) & \cong \mathbb{F} \otimes_{T_{V_{s}} D_{s}} T_{V_{s}} M \\
& \cong \mathbb{F} \otimes_{T_{\left(V_{s}, i\right)} D_{s}} T_{\left(V_{s}, i\right)} M \cong \mathbb{F} \otimes_{H^{*} V_{s}} T_{\left(V_{s}, i\right)} M .
\end{aligned}
$$

For the case $M=H^{*} V_{s}$, one verifies that $T_{\left(V_{s}, i\right)} H^{*} V_{s}$ identifies with $\mathbb{F}^{\operatorname{Aut}\left(V_{s}\right)} \otimes H^{*} V_{s}$ as an $H^{*} V_{s}$-algebra, from which the result follows.

Theorem 8.2.2 For $s \in \mathbb{N}$, the natural transformation

$$
\operatorname{Fix}_{S}\left(H^{*} V_{s} \otimes_{D_{s}} R_{S}(-)\right) \rightarrow 1 \text { น }
$$

of functors on $U$, which is adjoint to the canonical inclusion $R_{S}(-) \hookrightarrow D_{S} \otimes(-) \hookrightarrow$ $H^{*} V_{s} \otimes(-)$, is an isomorphism.

Moreover, the natural monomorphism $R_{S}(-) \hookrightarrow D_{s} \otimes-$ induces the canonical inclusion

$$
\operatorname{Fix}_{s}\left(H^{*} V_{s} \otimes_{D_{s}} R_{S}(-)\right) \cong 1_{\iota} \hookrightarrow T_{V_{s}}(-) \cong \operatorname{Fix}_{s}\left(H^{*} V_{s} \otimes_{D_{s}}\left(D_{s} \otimes-\right)\right) .
$$

Proof The natural monomorphism $\gamma_{s}: R_{S} \hookrightarrow \widetilde{R}_{S}$ is an isomorphism up to $\omega_{s}$-torsion, by Theorem 8.1 .5 , hence it suffices to prove the result with $\widetilde{R}_{s}$ in place of $R_{s}$, since Fix $_{S}$ annihilates $\omega_{s}$-torsion, by [12, Proposition 0.8]. The defining equalizer diagram for $\widetilde{R}_{S} M$ gives rise to an equalizer diagram in $H^{*} V_{s}-U$ :

$$
H^{*} V_{s} \otimes_{D_{s}} \widetilde{R}_{S} M \longrightarrow H^{*} V_{s} \otimes M \longrightarrow H^{*} V_{s} \otimes_{D_{s}} H^{*} V_{s} \otimes T_{V_{s}} M,
$$

since $H^{*} V_{s} \otimes_{D_{s}}(-)$ is exact.

The functor Fix $_{s}$ is exact, hence this gives the equalizer diagram in $U$ :

$\operatorname{Fix}_{S}\left(H^{*} V_{s} \otimes_{D_{s}} \widetilde{R}_{S} M\right) \longrightarrow \operatorname{Fix}_{s}\left(H^{*} V_{s} \otimes M\right) \Longrightarrow \operatorname{Fix}_{S}\left(H^{*} V_{s} \otimes_{D_{s}} H^{*} V_{s} \otimes T_{V_{s}} M\right)$.

There are natural isomorphisms $\operatorname{Fix}_{s}\left(H^{*} V_{s} \otimes M\right) \cong T_{V_{s}} M$ and

$$
\operatorname{Fix}_{s}\left(H^{*} V_{s} \otimes D_{s} H^{*} V_{s} \otimes T_{V_{s}} M\right) \cong \mathbb{F}^{\operatorname{Aut}\left(V_{s}\right)} \otimes T_{V_{s}} T_{V_{s}} M,
$$

obtained by viewing $H^{*} V_{s} \otimes_{D_{s}} H^{*} V_{s} \otimes T_{V_{s}} M$ as the tensor product over $H^{*} V_{s}$ of $H^{*} V_{s} \otimes_{D_{s}} H^{*} V_{s}$ and $H^{*} V_{s} \otimes T_{V_{s}} M$ and applying Lemma 8.2.1.

The equalizer diagram therefore identifies with

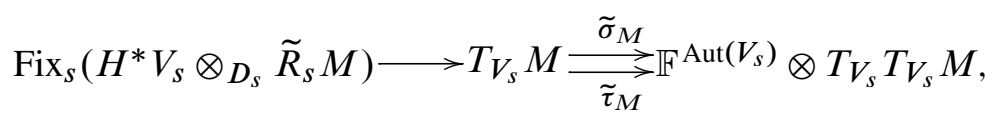

where $\widetilde{\sigma}_{M}:=\operatorname{Fix}_{S}\left(H^{*} V_{S} \otimes_{D_{s}} \sigma_{M}\right)$ and $\tilde{\tau}_{M}:=\operatorname{Fix}_{S}\left(H^{*} V_{S} \otimes_{D_{s}} \tau_{M}\right)$ are identified below. 
As in Remark 7.2.4, the result is a formal consequence of the following two points:

(1) the natural morphism $M \hookrightarrow T_{V_{s}} M$ equalizes the morphisms $\widetilde{\sigma}_{M}$ and $\tilde{\tau}_{M}$;

(2) $M \hookrightarrow T_{V_{s}} M$ is the equalizer of the diagram of unstable modules

$$
T_{V_{s}} M \rightrightarrows T_{V_{s}} T_{V_{s}} M
$$

which is obtained from $\tilde{\sigma}_{M}, \tilde{\tau}_{M}$ by composing with the surjection $\mathbb{F}^{\operatorname{Aut}\left(V_{s}\right)} \otimes$ $T_{V_{s}} T_{V_{s}} M \rightarrow T_{V_{s}} T_{V_{s}} M$ induced by the augmentation $\mathbb{F}^{\mathrm{Aut}\left(V_{s}\right)} \rightarrow \mathbb{F}$.

The identification of the morphisms $\widetilde{\sigma}_{M}$ and $\tilde{\tau}_{M}$ is a standard calculation with the $T$-functor; the precise form depends on the conventions used in the isomorphism $\operatorname{Fix}_{s}\left(H^{*} V_{s} \otimes_{D_{s}} H^{*} V_{s}\right) \cong \mathbb{F}^{\operatorname{Aut}\left(V_{s}\right)}$ of Lemma 8.2.1. The appropriate form can be deduced from the nillocalized case, as in Section 7.2, which leads to the following identifications.

For an automorphism $\alpha \in \operatorname{Aut}\left(V_{s}\right)$, the components

$$
T_{V_{s}} M \underset{\widetilde{\tau}_{M}^{\alpha}}{\stackrel{\widetilde{\sigma}_{M}^{\alpha}}{\longrightarrow}} T_{V_{s}} T_{V_{s}} M \cong T_{V_{s} \oplus V_{s}} M,
$$

of $\widetilde{\sigma}_{M}$ and $\tilde{\tau}_{M}$ indexed by $\alpha$ are induced by naturality of the $T$-functor by

$$
V_{s} \underset{1_{V_{s}} \Pi \alpha}{\stackrel{1_{V_{s}} \Pi 0}{\longrightarrow}} V_{s} \oplus V_{s}
$$

The two key points are established as in Remark 7.2.4: that $M$ lies in the equalizer follows since $\tilde{\sigma}_{M}, \tilde{\tau}_{M}$ are derived from the naturality with respect to $V$ of $T_{V}$; the second point follows by observing that the diagram

$$
M \longrightarrow T_{V_{s}} M \longrightarrow T_{V_{s} \oplus V_{s}} M
$$

is a split equalizer in unstable modules, by applying Lemma 7.2.2, where the morphisms $T_{V_{s}} M \rightrightarrows T_{V_{s} \oplus V_{s}} M$ are induced respectively by $1_{V_{s}} \prod 0: V_{s} \rightarrow V_{s} \oplus V_{s}$ and the diagonal $\Delta: V_{s} \rightarrow V_{s} \oplus V_{s}$.

The final statement has been established in the course of the proof.

\subsection{The Singer functor is a fully-faithful embedding}

Proposition 7.1.4 shows that the unit $1 \mathscr{F} \rightarrow \mathcal{R}_{s}^{0} \kappa_{s}$ of the adjunction $\kappa_{s} \dashv \mathcal{R}_{s}^{0}$ is a natural isomorphism. This section shows that Theorem 8.2.2 implies the analogous statement for the adjunction $R_{S} \dashv \mathfrak{Z}_{s}$; in particular, the functor $R_{S}: U \rightarrow D_{s}-\mathcal{U}$ is rigid, considered as a functor to unstable $D_{s}$-modules. 
Theorem 8.3.1 For $s \in \mathbb{N}$, the adjunction unit $1_{\vartheta} \rightarrow \mathfrak{Z}_{s} R_{s}$ is a natural isomorphism and the natural inclusions $R_{s} \hookrightarrow D_{s} \otimes(-) \hookrightarrow H^{*}\left(V_{s}\right) \otimes(-)$ in $D_{s}-U$ induce isomorphisms

$$
1_{u} \cong \mathfrak{Z}_{s} R_{s} \cong \mathfrak{Z}_{s}\left(D_{s} \otimes-\right) \cong \mathfrak{Z}_{s}\left(H^{*} V_{s} \otimes-\right) .
$$

In particular, $R_{s}$ induces a fully-faithful embedding $R_{s}: U \hookrightarrow D_{s}-U$.

Proof For the first statement, it suffices to prove that, for $M, N \in \mathrm{Ob} u$, the functor $R_{S}$ induces a natural isomorphism:

$$
\operatorname{Hom}_{u}(M, N) \longrightarrow \operatorname{Hom}_{D_{S}-\Omega}\left(R_{S} M, R_{S} N\right) \text {. }
$$

This is a monomorphism by Lemma 4.1.4; composing with the natural inclusion $R_{s} N \hookrightarrow D_{s} \otimes N \hookrightarrow H^{*} V_{s} \otimes N$, there is a natural monomorphism

$$
\begin{aligned}
\operatorname{Hom}_{D_{s}-\Omega}\left(R_{S} M, R_{S} N\right) & \hookrightarrow \operatorname{Hom}_{D_{s}-u}\left(R_{S} M, H^{*} V_{s} \otimes N\right) \\
& \cong \operatorname{Hom}_{H^{*} V_{s}-u}\left(H^{*} V_{s} \otimes_{D_{s}} R_{S} M, H^{*} V_{s} \otimes N\right) .
\end{aligned}
$$

By adjunction,

$\operatorname{Hom}_{H^{*} V_{S}-u}\left(H^{*} V_{s} \otimes_{D_{s}} R_{S} M, H^{*} V_{s} \otimes N\right) \cong \operatorname{Hom}_{U}\left(\operatorname{Fix}_{S}\left(H^{*} V_{s} \otimes_{D_{s}} R_{S} M\right), N\right)$,

and, by Theorem 8.2.2, $\operatorname{Fix}_{S}\left(H^{*} V_{s} \otimes_{D_{s}} R_{s} M\right) \cong M$. Thus, there are natural monomorphisms

$$
\begin{aligned}
\operatorname{Hom}_{D_{S}-u}\left(R_{S} M, R_{S} N\right) \hookrightarrow \operatorname{Hom}_{D_{s}-u}\left(R_{S} M, D_{s} \otimes N\right) & \hookrightarrow \operatorname{Hom}_{D_{S}-u}\left(R_{S} M, H^{*} V_{s} \otimes N\right) \\
& \cong \operatorname{Hom}_{U}(M, N) .
\end{aligned}
$$

The composite with the natural inclusion $\operatorname{Hom}_{U}(M, N) \hookrightarrow \operatorname{Hom}_{D_{S}-u}\left(R_{S} M, R_{S} N\right)$ is the identity, which establishes the natural isomorphisms.

The property of the adjunction unit is a formal consequence.

\section{The functors $\mathfrak{Z}_{s}, Q_{s}$ and $\mathbf{F i x} s$}

The purpose of this section is to provide a better understanding of the right adjoint $\mathfrak{Z}_{s}$ to the Singer functor $R_{S}$, in particular its relationship with the indecomposables functor $Q_{s}$ and with the functor $\operatorname{Fix}_{S}\left(H^{*} V_{s} \otimes_{D_{s}}-\right)$. 


\subsection{The Singer functor $R_{s}$ and the indecomposables $Q_{s}$}

In [3, Section 3] and [4, Section 3], Dwyer and Wilkerson studied a linear operation constructed from the Steenrod total power $\mathrm{St}_{1}$. This is related to the natural transformation defined below (defined for arbitrary $s$ ), where forget $D_{s}: D_{s}-U$ is the forgetful functor.

Definition 9.1.1 For $s \in \mathbb{N}$, let $\varepsilon_{s}: R_{S}$ forget $_{s} \rightarrow 1_{D_{s}-\Omega}$ be the natural transformation defined on $M \in \mathrm{Ob} D_{s}-U$ as the composite

$$
R_{S} M \hookrightarrow D_{s} \otimes M \rightarrow M
$$

of the canonical inclusion followed by the product.

Proposition 9.1.2 The natural transformation forget $_{s} \rightarrow Q_{s}$ of functors from $D_{s}-\mathcal{U}$ to $U$ induces a factorization

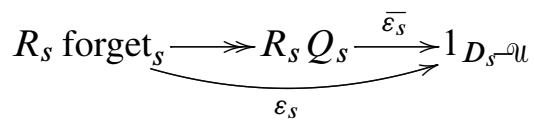

of endofunctors of $D_{s}-U$.

Proof The proof proceeds by reduction to the behaviour on $D_{s}$. For $M \in \mathrm{Ob} D_{s}-\mathcal{U}$, there is an exact sequence of unstable modules

$$
\overline{D_{s}} \otimes M \rightarrow M \rightarrow Q_{s} M \rightarrow 0,
$$

where the first morphism is induced by multiplication. The functor $R_{S}$ is exact, hence, by naturality of $\varepsilon_{s}$, it suffices to prove that the composite morphism

$$
R_{s}\left(\overline{D_{s}} \otimes M\right) \stackrel{\varepsilon_{s}}{\longrightarrow} \overline{D_{s}} \otimes M \rightarrow M
$$

is zero.

There is a natural isomorphism $R_{S}\left(\overline{D_{S}} \otimes M\right) \cong R_{S}\left(\overline{D_{S}}\right) \otimes{ }_{D_{S}} R_{S} M$, by the monoidal property of $R_{s}$ (see Proposition 4.1.3) and, with respect to this, the above composite is induced by the tensor product over $D_{s}$ of $\varepsilon_{s}: R_{s} \overline{D_{s}} \rightarrow \overline{D_{s}}$ and $\varepsilon_{s}: R_{s} M \rightarrow M$. Therefore, to prove the result, it is sufficient to show that the morphism $\varepsilon_{s}: R_{S} \overline{D_{s}} \rightarrow \overline{D_{S}}$ is trivial.

This can be proved directly, generalizing [3, Lemma 3.3(ii)], by reducing to the case $s=1$, using the fact [12] that $\mathrm{St}_{s}$ is the $s$-fold iterate of $\mathrm{St}_{1}$.

An alternative method is to use passage to nillocalization. Since $R_{S}$ preserves reduced objects and $\overline{D_{s}}$ is reduced, it is sufficient to prove that the induced morphism 
$\mathfrak{l}_{D_{s}} R_{S}\left(\overline{D_{S}}\right) \rightarrow \mathfrak{l}_{D_{s}} \overline{D_{S}}$ is trivial. The natural transformation $\varepsilon_{s}$ corresponds to the natural transformation

$$
\kappa_{s} \Omega^{\mathfrak{g}\left(D_{s}\right)} \rightarrow 1_{\mathscr{F} \mathfrak{g}\left(D_{s}\right)}
$$

of endofunctors of $\mathscr{F}^{\mathfrak{g}}\left(D_{s}\right)$ given by the composite

$$
\kappa_{S} \Omega^{\mathfrak{g}\left(D_{s}\right)} \hookrightarrow \iota^{\mathfrak{g}\left(D_{s}\right)} \Omega^{\mathfrak{g}\left(D_{s}\right)} \rightarrow 1_{\mathscr{F} \mathfrak{g}\left(D_{s}\right)}
$$

induced by the inclusion $\kappa_{S} \hookrightarrow \iota^{\mathfrak{g}\left(D_{s}\right)}$ and the counit of the $\iota^{\mathfrak{g}\left(D_{s}\right)} \dashv \Omega^{\mathfrak{g}\left(D_{s}\right)}$ adjunction. For $G \in \mathrm{Ob} \mathscr{F}^{\mathfrak{g}}\left(D_{s}\right)$ and $(V, W) \in \mathrm{Ob}^{f} f / \mathfrak{g}\left(D_{s}\right)$, by using the explicit form of the $\iota^{\mathfrak{g}\left(D_{s}\right)} \dashv \Omega^{\mathfrak{g}\left(D_{s}\right)}$ adjunction counit, this identifies as the surjection

$$
\bigoplus_{\operatorname{codim} U \leq s} G(W, U) \rightarrow G(V, W)
$$

given by projection onto the summand indexed by $U=W$ followed by the morphism $G(W, W) \rightarrow G(V, W)$ induced by $(W, W) \rightarrow(V, W)$ in $\mathscr{V}^{f} / \mathfrak{g}\left(D_{s}\right)$.

Now, $\mathfrak{l}_{D_{s}} D_{s}$ is the constant functor $\mathbb{F} \in \mathscr{F}^{\mathfrak{g}}\left(D_{s}\right)$ and the augmentation ideal gives the subfunctor $\mathfrak{l}_{D_{s}} \overline{D_{s}}$ :

$$
(V, W) \mapsto \begin{cases}\mathbb{F} & V \neq W \\ 0 & V=W\end{cases}
$$

The result follows.

The following corollary is formal.

Corollary 9.1.3 For $s \in \mathbb{N}$, the natural transformation $\zeta_{s}: Q_{s} \rightarrow \mathfrak{Z}_{s}$ adjoint to $\overline{\varepsilon_{s}}: R_{s} Q_{s} \rightarrow 1_{D_{s}-\Omega}$, fits into a commutative diagram:

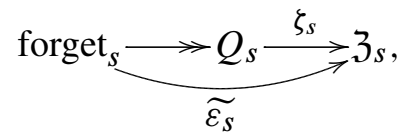

where $\widetilde{\varepsilon_{s}}$ : forget $_{s} \rightarrow \mathfrak{Z}_{s}$ is adjoint to $\varepsilon_{s}: R_{s}$ forget $_{s} \rightarrow 1_{D_{S}} U$.

The following natural transformation is used in Theorem 9.2.2.

Lemma 9.1.4 For $s \in \mathbb{N}$, the functor $Q_{s}$ applied to the adjunction counit $R_{s} \mathfrak{Z}_{s} \rightarrow 1_{D_{s}}$ u induces a natural transformation $\xi_{s}: \Phi^{s} \mathfrak{Z}_{s} \rightarrow Q_{s}$.

Proof This is an immediate consequence of the natural isomorphism $Q_{s} R_{S} \cong \Phi^{s}$ of Proposition 4.1.3. 


\subsection{The relationship between $Q_{s}$ and $\mathfrak{Z}_{s}$}

In order to understand the relationship between $Q_{s}$ and $\mathfrak{Z}_{s}$, further information on the behaviour of $\mathfrak{Z}_{s}$ is required. Recall that the category $D_{s}-U$ has enough injectives [12; $7 ; 15]$.

Lemma 9.2.1 If $I \in \mathrm{Ob} D_{s}-\mathcal{U}$ is injective, then the natural morphism $\zeta_{s}: Q_{s} I \rightarrow \mathfrak{Z}_{s} I$ is surjective.

Proof It suffices to show that the morphism forget $I \rightarrow \mathfrak{Z}_{s} I$ of Corollary 9.1.3 is surjective. For $k \in \mathbb{N}$, there is a canonical embedding $R_{s} F(k) \hookrightarrow D_{s} \otimes F(k)$ in $D_{s}-U$, and hence, by injectivity of $I$, a surjection

$$
\operatorname{Hom}_{D_{S}-\Omega}\left(D_{s} \otimes F(k), I\right) \rightarrow \operatorname{Hom}_{D_{S}-\Omega}\left(R_{S} F(k), I\right) .
$$

This corresponds to the degree $k$ part of the morphism forget $I \rightarrow \mathfrak{Z}_{s} I$, which is therefore surjective.

Recall that $\lambda: \Phi \rightarrow 1_{u}$ is the natural transformation induced by $\mathrm{Sq}_{0}$, which induces $\lambda^{s}: \Phi^{s} \rightarrow 1_{\varkappa}$ by iteration, for $s \in \mathbb{N}$. The natural transformation $\xi_{s}$ was introduced in Lemma 9.1.4.

\section{Theorem 9.2.2 For $s \in \mathbb{N}$,}

(1) the composite $\Phi^{s} Q_{s} \stackrel{\Phi^{s} \zeta_{s}}{\longrightarrow} \Phi^{s} \mathfrak{Z}_{s} \stackrel{\xi_{s}}{\longrightarrow} Q_{s}$ is the natural transformation $\lambda_{Q_{s}}^{s}$;

(2) the composite $\Phi^{s} \mathfrak{Z}_{s} \stackrel{\xi_{s}}{\longrightarrow} Q_{s} \stackrel{\zeta_{s}}{\longrightarrow} \mathfrak{Z}_{s}$ is the natural transformation $\lambda_{\mathfrak{Z}_{s}^{s}}$.

In particular, $\zeta_{s}: Q_{s} \rightarrow \mathfrak{Z}_{s}$ and $\xi_{s}: \Phi^{s} \mathfrak{Z}_{s} \rightarrow Q_{s}$ are isomorphisms up to nilpotent unstable modules and the functor $\mathfrak{Z}_{s}$ sends nilpotents to nilpotents.

Proof The first natural transformation is given by applying the functor $Q_{s}$ to the composite $R_{s} Q_{s} \rightarrow R_{s} \mathfrak{Z}_{s} \rightarrow 1_{D_{S}-\Omega}$. The identification follows from the fact that, modulo decomposables, $\mathrm{St}_{s}$ identifies with the linear map $\left(\mathrm{Sq}_{0}\right)^{s}$.

The functor $\mathfrak{Z}_{s}$ is left exact and $D_{s}-\mathcal{U}$ has enough injectives, hence it suffices to show that the natural transformation identifies with $\lambda_{\mathfrak{Z}_{s}}^{s}$ when evaluated on any injective object $I$.

Consider the composite

$$
\Phi^{s} Q_{s} \underset{\underset{\Phi^{s} \zeta_{s}}{\longrightarrow}}{\lambda_{Q_{s}}^{s}} \Phi^{s} \mathfrak{Z}_{s} I \underset{\xi_{s}}{\longrightarrow} Q_{\lambda_{\mathfrak{Z} s}^{s}} I \stackrel{\zeta_{s}}{\longrightarrow} \mathfrak{Z}_{s} I .
$$


Here the surjection $\Phi^{s} \zeta_{s}$ is given by Lemma 9.2.1, since the functor $\Phi^{s}$ is exact, and the identification of $\lambda_{Q_{s}}^{s}$ follows from the first part of the theorem.

To prove the result, by surjectivity of $\Phi^{s} \zeta_{s}$, it suffices to show that the composites $\zeta_{s} \xi_{s}\left(\Phi^{s} \zeta_{s}\right)$ and $\lambda_{\mathcal{Z}_{s}}^{s}\left(\Phi^{s} \zeta_{s}\right)$ coincide evaluated on $I$. This follows from the naturality of $\lambda^{s}$, which gives the commutative diagram of natural transformations

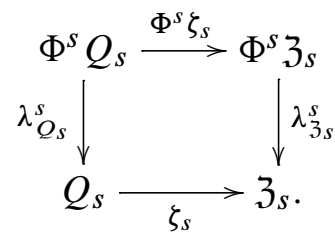

The final statements are immediate consequences of these identifications, since the functor $Q_{s}$ sends objects with nilpotent underlying unstable module to nilpotent unstable modules.

In the following, $\mathcal{R}_{s}^{0}$ is the functor of Proposition 6.2.1.

Corollary 9.2.3 For $s \in \mathbb{N}$,

(1) there are natural isomorphisms

(a) $\mathfrak{Z}_{s} \mathfrak{r}_{D_{s}} \cong r \mathcal{R}_{s}^{0}: \mathscr{F}^{g}\left(D_{s}\right) \rightarrow \mathcal{U}$,

(b) $l \mathfrak{Z}_{s} \cong \mathcal{R}_{s}^{0} \mathfrak{l}_{D_{s}}: D_{s}-\mathcal{U} \rightarrow \mathscr{F}$,

and, in particular, the functor $l \mathfrak{Z}_{s}$ is exact;

(2) for $N \in \mathrm{Ob} D_{s}-U$ which is reduced, $\mathfrak{Z}_{s} N=0$ if and only if $Q_{s} N$ is nilpotent.

Proof The first isomorphism is a formal consequence of Corollary 8.1.6. Namely, $\mathfrak{Z}_{s} \mathfrak{r}_{D_{s}}$ is right adjoint to $\mathfrak{l}_{D_{s}} R_{s}$, which is naturally equivalent to $\kappa_{s} l$, by Corollary 8.1.6. The latter is left adjoint to $r \mathcal{R}_{s}^{0}$, by Proposition 7.1.4.

Precomposing with $\mathfrak{l}_{D_{s}}$ and postcomposing with $l$ gives a natural isomorphism $l \mathfrak{Z} \mathfrak{r}_{D_{s}} \mathfrak{l}_{D_{s}} \cong \mathcal{R}_{s}^{0} \mathfrak{l}_{D_{s}}$. The natural transformation $1_{D_{s}-\boldsymbol{U}} \rightarrow \mathfrak{r}_{D_{s}} \mathfrak{l}_{D_{s}}$ is an isomorphism modulo nilpotent objects, hence, by Theorem 9.2.2, the induced natural transformation $l \mathfrak{Z}_{s} \longrightarrow l \mathfrak{Z}_{D_{S}} \mathfrak{l}_{D_{s}}$ is an isomorphism; this gives the second natural isomorphism.

Suppose now that $N \in \mathrm{Ob} D_{s}-\mathcal{U}$ is a reduced object; hence, by Proposition 4.1.6, $\mathfrak{Z}_{s} N$ is reduced. Thus, $\mathfrak{Z}_{s} N=0$ if and only if $l \mathfrak{Z}_{s} N=0$, which is equivalent to $l Q_{s} N=0$, by Theorem 9.2.2.

Corollary 9.2.4 If $M \in \mathrm{Ob} D_{s}-\mathcal{U}$ is a reduced object, then $\mathfrak{Z}_{s}\left(\omega_{s} M\right)=0$. 
Proof The $D_{s}$-module $\omega_{s} M$ is a submodule of $M$, hence is reduced, so it suffices to show that $Q_{s}\left(\omega_{s} M\right)$ is nilpotent. The short exact sequence

$$
0 \rightarrow \omega_{s} M \rightarrow M \rightarrow M / \omega_{s} M \rightarrow 0
$$

in $D_{s}-U$ induces an exact sequence

$$
Q_{s}\left(\omega_{s} M\right) \rightarrow Q_{s} M \rightarrow Q_{s}\left(M / \omega_{s} M\right) \rightarrow 0,
$$

which is short exact modulo nilpotent unstable modules, since by Proposition 6.2.1 $l Q_{s}$ is exact. The surjection $Q_{s} M \rightarrow Q_{s}\left(M / \omega_{s} M\right)$ is an isomorphism, so the result follows.

Example 9.2.5 For $s \in \mathbb{N}, N \in \mathrm{Ob} U$ and $M \in \mathrm{Ob} D_{s}-\mathcal{U}$ which is reduced,

$$
\operatorname{Hom}_{D_{S}-\Omega}\left(R_{S} N, \omega_{S} M\right)=0 .
$$

For example, take $M=D_{s}$.

\subsection{The relationship between $\mathfrak{Z}_{s}$ and $\operatorname{Fix}_{s}\left(H^{*} V_{s} \otimes_{D_{s}}-\right)$}

It is interesting to have a criterion for the counit $R_{s} \mathfrak{Z}_{s} M \rightarrow M$ (for $M \in \mathrm{Ob} D_{s}-\mathcal{U}$ ) to be an isomorphism. By Theorem 8.3.1, if $M \cong R_{s} N$ for some $N \in \mathrm{Ob} U$, then $N \cong \mathfrak{Z}_{s} M$.

Proposition 9.3.1 For $s \in \mathbb{N}$, there is a natural transformation

$$
\mathfrak{Z}_{s} \rightarrow \operatorname{Fix}_{s}\left(H^{*} V_{s} \otimes_{D_{s}}(-)\right)
$$

of functors from $D_{s}-\mathcal{U}$ to $U$.

Moreover, if $M \in \mathrm{Ob} D_{s}-\mathcal{U}$ such that the counit $R_{s} \mathfrak{Z}_{s} M \rightarrow M$ is an isomorphism, then

$$
\mathfrak{Z}_{S} M \rightarrow \operatorname{Fix}_{S}\left(H^{*} V_{s} \otimes_{D_{s}} M\right)
$$

is an isomorphism.

Proof The natural transformation is given by applying the functor $\operatorname{Fix}_{s}\left(H^{*} V_{s} \otimes_{D_{s}}(-)\right)$ to the counit $R_{s} \mathfrak{Z}_{s} \rightarrow 1_{D_{s}-U}$, using the isomorphism of Theorem 8.2.2. If the counit is an isomorphism, then so is the induced natural morphism.

Remark 9.3.2 (1) Composition with the natural transformation forget $_{s} \rightarrow \mathfrak{Z}_{s}$ of Corollary 9.1.3 induces a natural morphism

$$
\text { forget }_{s} \rightarrow \operatorname{Fix}_{s}\left(H^{*} V_{s} \otimes_{D_{s}}(-)\right) \text {. }
$$

This is induced by the natural transformation of Proposition 2.4.1. 
(2) The natural transformation $\mathfrak{Z}_{s} \rightarrow \operatorname{Fix}_{s}\left(H^{*} V_{s} \otimes_{D_{s}}(-)\right)$ corresponds to the natural transformation $\mathcal{R}_{s}^{0} \rightarrow \Psi_{s} \operatorname{Ind}_{s}$ of functors from $\mathscr{F}_{\mathfrak{g}}\left(D_{s}\right)$ to $\mathscr{F}_{\mathcal{F}}$ which is given on $G \in \mathrm{Ob} \mathscr{F}_{\mathfrak{g}}\left(D_{s}\right)$ by $\mathcal{R}_{s}^{0} G(V)=G(V, V) \rightarrow \Psi_{s} \operatorname{Ind}_{s} G(V)=G\left(V \oplus V_{s}, V\right)$, induced by $(V, V) \rightarrow\left(V \oplus V_{s}, V\right)$ (cf Propositions 6.3.2 and 6.2.1).

For $s>1$, it is straightforward to see that $\mathfrak{Z}_{s} M \rightarrow \operatorname{Fix}_{s}\left(H^{*} V_{s} \otimes_{D_{s}} M\right)$ being an isomorphism does not imply in general that the counit is an isomorphism. However, in the case $s=1$, one has the following.

Theorem 9.3.3 For $M \in \mathrm{Ob} D_{1}-U$ such that the underlying unstable module is reduced, the following conditions are equivalent:

(1) the counit $R_{1} \mathfrak{Z}_{1} M \rightarrow M$ is an isomorphism;

(2) the natural morphism $\mathfrak{Z}_{1} M \rightarrow$ Fix $_{1} M$ is an isomorphism.

Proof Proposition 9.3.1 gives (1) $\Rightarrow$ (2).

For the converse, consider the exact sequence in $D_{1}-U$

$$
0 \rightarrow \mathrm{Ker} \rightarrow R_{1} \mathfrak{Z}_{1} M \rightarrow M \rightarrow \text { Coker } \rightarrow 0 .
$$

The hypothesis (2) implies that $\operatorname{Fix}_{1}\left(R_{1} \mathfrak{Z}_{1} M \rightarrow M\right)$ is an isomorphism. Thus, by [12, Proposition 0.8], both Ker and Coker are $\omega_{1}$-torsion. However, by construction, Ker is a subobject of $R_{1} \mathfrak{Z}_{1} M$, which is $\omega_{1}$-torsion free, hence Ker $=0$ and $R_{1} \mathfrak{Z}_{1} M \hookrightarrow M$ is a monomorphism with $\omega_{1}$-torsion cokernel.

By hypothesis, $M$ is reduced, hence $\mathfrak{Z}_{1} M \cong \operatorname{Fix}_{1} M$ is reduced. Theorem 8.1.5 implies that $R_{1} \mathfrak{Z}_{1} M$ is $\omega_{1}$-closed. Hence, to complete the proof, it suffices to show that $M$ is $\omega_{1}$-torsion free. Consider the submodule $A:=\operatorname{Ann}_{\omega_{1}} M \subset M$, so that $A$ is in the image of triv ${ }_{1}: \mathcal{U} \rightarrow D_{1}-\mathcal{U}$. The module $M$ is $\omega_{1}$-torsion free if and only if $A=0$.

Applying the functor $\mathfrak{Z}_{1}$ yields a monomorphism

$$
\widetilde{\Phi} A \cong \mathfrak{Z}_{1} A \hookrightarrow \mathfrak{Z}_{1} M \cong \operatorname{Fix}_{1} M,
$$

where the first isomorphism is given by Proposition 4.1.8. By naturality of $\mathfrak{Z}_{1} \rightarrow$ Fix $_{1}$, this factors across Fix $_{1} A$, which is trivial (since $A$ is $\omega_{1}$-torsion). Thus $\widetilde{\Phi} A=0$. However, $A$ is a reduced unstable module, since it is a submodule of $M$, hence $A$ must be zero, as required. 


\section{Appendix A General results}

\section{A.1 The right adjoint to $\Phi$ on categories of modules}

Proposition A.1.1 For $K$ an unstable algebra, $\widetilde{\Phi}$ induces a functor $\widetilde{\Phi}: \Phi K-U \rightarrow K-U$ which is right adjoint to $\Phi: K-\vartheta \rightarrow \Phi K-\vartheta$.

Proof The functor $\Phi$ commutes with tensor products, hence the adjunction counit $\Phi \widetilde{\Phi} \rightarrow 1_{\imath}$ induces a natural morphism

$$
\widetilde{\Phi} M \otimes \widetilde{\Phi} N \rightarrow \widetilde{\Phi}(M \otimes N)
$$

for $M, N \in \mathrm{Ob} u$. Thus, if $M \in \mathrm{Ob} \Phi K-\vartheta, \widetilde{\Phi} M$ is an object of $K-\vartheta$ with respect to the structure morphism:

$$
K \otimes \widetilde{\Phi} M \cong \widetilde{\Phi} \Phi K \otimes \widetilde{\Phi} M \rightarrow \widetilde{\Phi}(\Phi K \otimes M) \rightarrow \widetilde{\Phi} M
$$

where the last morphism is induced by the structure morphism of $M$. (By construction, this is a morphism of $U$; the associativity and unit axioms are straightforward verifications.)

By definition, for $N \in \mathrm{Ob} K-\vartheta, \operatorname{Hom}_{K-U}(N, \widetilde{\Phi} M)$ is the equalizer of

$$
\operatorname{Hom}_{u}(N, \widetilde{\Phi} M) \rightrightarrows \operatorname{Hom}_{u}(K \otimes N, \widetilde{\Phi} M) \text {. }
$$

By adjunction, this is equivalent to the diagram

$$
\operatorname{Hom}_{u}(\Phi N, M) \rightrightarrows \operatorname{Hom}_{u}(\Phi K \otimes \Phi N, M) \text {. }
$$

A simple verification shows that this corresponds to the equalizer diagram defining $\operatorname{Hom}_{\Phi K-U}(\Phi N, M)$, which completes the proof.

\section{A.2 Formal results for endofunctors of $U$}

The following results explain how to study exact endofunctors of the category $u$ via passage to $U / \mathcal{N} i l$.

Lemma A.2.1 Let $\Theta: ~ U \rightarrow \mho$ be an exact functor which preserves the subcategory $\mathcal{N} i l$, then

(1) $U \stackrel{\Theta}{\longrightarrow} \cup \stackrel{l}{\rightarrow} \cup / \mathcal{N} i l$ induces an exact functor $\bar{\Theta}: \cup / \mathcal{N} i l \rightarrow q / \mathcal{N} i l$ such that $\bar{\Theta} l \cong l \Theta$

(2) if, moreover, $\Theta$ preserves the class of nilclosed unstable modules, then there is a natural isomorphism $\Theta r l \cong r \bar{\Theta} l$. In particular, $\bar{\Theta}$ determines the restriction of $\Theta$ to the full subcategory of nilclosed unstable modules. 
Recall that $\mathcal{U} / \mathcal{N} i l$ is equivalent to the category of analytic functors, $\mathscr{F}_{\omega} \omega$. The category $\mathscr{F}_{\omega} \omega$ is locally Noetherian [19, Proposition 5.3.3], hence any coproduct of injective cogenerators of $\mathscr{F}_{\omega} \omega$ of the form $I_{V}$ is injective in $\mathscr{F}_{\omega}$ and any analytic functor admits an injective resolution in which each term is of the form $\bigoplus_{\alpha} I_{V_{\alpha}}$. This implies the following result.

Lemma A.2.2 Let $\Theta_{1}, \Theta_{2}: \mathscr{F}_{\omega} \rightarrow \mathscr{F}_{\omega}$ be two exact functors which commute with arbitrary coproducts. If the restrictions $\Theta_{1}, \Theta_{2}$ to the full subcategory with objects $\left\{I_{V_{s}} \mid s \in \mathbb{N}\right\}$ are naturally isomorphic, then $\Theta_{1}$ and $\Theta_{2}$ are naturally isomorphic.

\section{A.3 Preservation of reduced unstable modules}

Lemma A.3.1 Let $\gamma: G_{1} \hookrightarrow G_{2}$ be a natural monomorphism of endofunctors of $\mathcal{U}$ such that

(1) $G_{1}$ is exact and $G_{2}$ is left exact;

(2) $\gamma_{M}$ is an isomorphism if $M$ is a nilclosed unstable module.

Then $\gamma_{N}: G_{1} N \rightarrow G_{2} N$ is an isomorphism if $N$ is a reduced unstable module.

Proof Consider a reduced unstable module $N$ and the associated short exact sequence of unstable modules $0 \rightarrow N \rightarrow r l N \rightarrow(r l N) / N \rightarrow 0$. The natural monomorphism $\gamma$ induces a commutative diagram in $U$

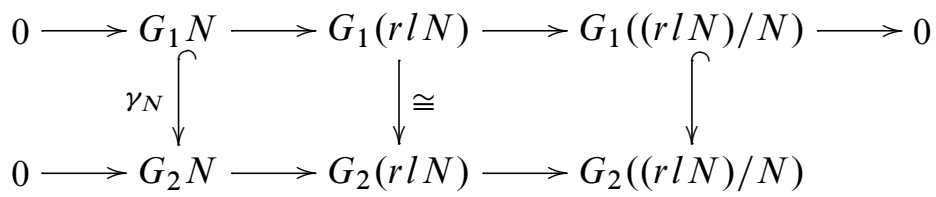

in which the rows are exact and the middle vertical morphism is an isomorphism, since $r l N$ is nilclosed. The result follows from the five-lemma.

\section{References}

[1] J F Adams, C W Wilkerson, Finite $H$-spaces and algebras over the Steenrod algebra, Ann. of Math. 111 (1980) 95-143 MR558398

[2] A Djament, Foncteurs en grassmanniennes, filtration de Krull et cohomologie des foncteurs, Mém. Soc. Math. Fr. (2007) xxii+213 pp. MR2482711

[3] W G Dwyer, C W Wilkerson, Smith theory revisited, Ann. of Math. 127 (1988) 191198 MR924676 
[4] W G Dwyer, C W Wilkerson, Smith theory and the functor T, Comment. Math. Helv. 66 (1991) 1-17 MR1090162

[5] W G Dwyer, C W Wilkerson, Kähler differentials, the $T$-functor, and a theorem of Steinberg, Trans. Amer. Math. Soc. 350 (1998) 4919-4930 MR1621741

[6] P Gabriel, Des catégories abéliennes, Bull. Soc. Math. France 90 (1962) 323-448 MR0232821

[7] H-W Henn, Commutative algebra of unstable $K$-modules, Lannes' $T$-functor and equivariant mod-p cohomology, J. Reine Angew. Math. 478 (1996) 189-215 MR1409058

[8] H-W Henn, J Lannes, L Schwartz, The categories of unstable modules and unstable algebras over the Steenrod algebra modulo nilpotent objects, Amer. J. Math. 115 (1993) 1053-1106 MR1246184

[9] H-W Henn, J Lannes, L Schwartz, Localizations of unstable A-modules and equivariant mod p cohomology, Math. Ann. 301 (1995) 23-68 MR1312569

[10] J Lannes, Sur les espaces fonctionnels dont la source est le classifiant d'un p-groupe abélien élémentaire, Inst. Hautes Études Sci. Publ. Math. (1992) 135-244 MR1179079

[11] J Lannes, S Zarati, Sur les foncteurs dérivés de la déstabilisation, Math. Z. 194 (1987) 25-59 MR871217

[12] J Lannes, S Zarati, Théorie de Smith algébrique et classification des $H^{*} V-U-$ injectifs, Bull. Soc. Math. France 123 (1995) 189-223 MR1340287

[13] J Lannes, S Zarati, Tor- et Ext-dimensions des $H^{*} V-A$-modules instables qui sont de type fini comme $H^{*} V$-modules, from: "Algebraic topology: New trends in localization and periodicity", (C Broto, C Casacuberta, G Mislin, editors), Progr. Math. 136, Birkhäuser, Basel (1996) 241-253 MR1397735

[14] D Mekkia, Sur les catégories $K-U$, Thèse de troisième cycle, Université de Tunis II (1994)

[15] D M Meyer, Injective objects in categories of unstable $K$-modules, Bonner Mathematische Schriften 316, Universität Bonn Mathematisches Institut (1999) MR1932593

[16] M D Neusel, L Smith, Invariant theory of finite groups, Mathematical Surveys and Monographs 94, American Mathematical Society (2002) MR1869812

[17] G M L Powell, Module structures and the derived functors of iterated loop functors on unstable modules over the Steenrod algebra, J. Pure Appl. Algebra 214 (2010) 1435-1449 MR2593673

[18] D L Rector, Noetherian cohomology rings and finite loop spaces with torsion, J. Pure Appl. Algebra 32 (1984) 191-217 MR741965

[19] L Schwartz, Unstable modules over the Steenrod algebra and Sullivan's fixed point set conjecture, University of Chicago Press (1994) MR1282727 
[20] W M Singer, The construction of certain algebras over the Steenrod algebra, J. Pure Appl. Algebra 11 (1977/78) 53-59 MR0467746

[21] W M Singer, On the localization of modules over the Steenrod algebra, J. Pure Appl. Algebra 16 (1980) 75-84 MR549705

[22] W M Singer, A new chain complex for the homology of the Steenrod algebra, Math. Proc. Cambridge Philos. Soc. 90 (1981) 279-292 MR620738

[23] W M Singer, Invariant theory and the lambda algebra, Trans. Amer. Math. Soc. 280 (1983) 673-693 MR716844

[24] C Wilkerson, Classifying spaces, Steenrod operations and algebraic closure, Topology 16 (1977) 227-237 MR0442932

[25] C Wilkerson, A primer on the Dickson invariants, from: "Proceedings of the Northwestern Homotopy Theory Conference", (H R Miller, S B Priddy, editors), Contemp. Math. 19, Amer. Math. Soc., Providence, RI (1983) 421-434 MR711066

Laboratoire Angevin de Recherche en Mathématiques, UMR 6093

Faculté des Sciences, Université d'Angers

2 Boulevard Lavoisier, 49045 Angers, France

Geoffrey.Powell@math.cnrs.fr

Received: 6 December 2010 Revised: 5 July 2012 